Begründung etwa in gewässerreichen Niederungsgebieten in Betracht kommen könne. ${ }^{75}$

\4a Abs. 2 PflSchAnwV-E sieht zudem die Möglichkeit einer behördlichen Ausnahmeerteilung von den Abstandsgeboten des Absatzes 1 Satz 1 und 2 zur Abwendung erheblicher wirtschaftlicher Schäden oder zum Schutz der heimischen Tier- und Pflanzenwelt, insbesondere vor invasiven Arten, vor.

\section{Weiteres Verfahren}

Im April 2021 wird die erste Lesung des Gesetzentwurfs zur Änderung des BNatSchG im Deutschen Bundestag stattfinden. Eine Stellungnahme des Bundesrates sowie die Gegenäußerung des Bundesregierung ist bereits erfolgt. ${ }^{76}$ Viel Zeit bleibt nicht mehr, aber es wäre ein Erfolg für die Bundesregierung und die Koalitionsfraktionen, dieses wichtige umweltpolitische Projekt noch in dieser Legislaturperiode umzusetzen.

Die Verordnung war aufgrund der in ihr enthaltenen Anwendungsbeschränkungen für glyphosathaltige Pflanzen- schutzmittel nun gemäß der Richtlinie (EU) 2015/1535 zunächst der Europäischen Kommission zu notifizieren, die diese unter unionsrechtlichen Aspekten zu prüfen hat und dazu eine Stellungnahme abgeben kann. Unter Berücksichtigung der durch die Notifizierung ausgelösten dreimonatigen „Stillhaltepflicht“ für das nationale Regelungsgebungsverfahren wird der Bundesrat frühestens im Mai 2021 über die für ihr Zustandekommen erforderliche Erteilung seiner Zustimmung zu der Verordnung entscheiden können.

75) Fünfte Verordnung zur Änderung der Pflanzenschutz-Anwendungsverordnung (Fn. 3), S. 14; diese Möglichkeit wird auch im Aktionsprogramm Insektenschutz (Fn. 6), S. 36, bereits angedacht. 76) BT-Drs. 19/28182 (Fn. 48).

77) Richtlinie 2015/1535 des Europäischen Parlaments und des Rates v. 9.9.2015 über ein Informationsverfahren auf dem Gebiet der technischen Vorschriften und der Vorschriften für die Dienste der Informationsgesellschaft, ABl. L 241 v. 17.9.2015, S. 1.

\title{
https://doi.org/10.1007/s10357-021-3827-1
}

\section{Ökonomische Instrumente der Phosphor-Governance}

\author{
Unter Berücksichtigung der Klima- und Biodiversitätsziele aus Paris-Abkommen und Biodiversitätskonvention
}

\section{Beatrice Garske/Felix Ekardt*}

○ Springer-Verlag GmbH Deutschland, ein Teil von Springer Nature 2021

Phosphor (P) ist eine lebensnotwendige und nicht-substituierbare Ressource für die landwirtschaftliche Erzeugung. Um die Nahrungsmittelversorgung sicherzustellen und gleichzeitig düngungsbezogene Umweltschäden zu vermeiden, bedarf es eines nachhaltigen P-Managements. Zwar werden P-bezogene Problemlagen teilweise durch das bestehende Agrar- und Umweltordnungsrecht adressiert (NuR 2018, 73ff.), allerdings mangelt es der gegenwärtigen Regulierung an einer ausreichenden Steuerungswirkung gemessen am Ziel eines nachhaltigeren P-Einsatzes. Zudem ist der bestehende Rechtsrahmen stark durch ordnungsrechtliche Detailregelungen geprägt und leidet unter Steuerungsproblemen wie Vollzugsdefiziten, Rebound- und Verlagerungseffekten. Wie diese Probleme mit Hilfe ökonomischer Instrumente überwunden werden könnten, ist Gegenstand des vorliegenden Beitrags. Ausgegangen wird dabei von zwei ökonomischen Instrumenten, mit denen zunächst die Einhaltung des Pariser Klimazieles und der Aichi-Ziele zur Biodiversitätskonvention als verbindliche globale Umweltziele sichergestellt wird: ein PrimärenergieEmissionshandel und ein Emissionshandel für tierische Produkte. Es wird analysiert, wie diese beiden Instrumente auf eine nachhaltige Landwirtschaft und auf die P-bezogenen Problemlagen wirken und inwiefern weiterer Ergänzungsbedarf konkret für P besteht.

\section{Problemstellung, Grundlagen, Methodik und Ziele eines nachhaltigen P-Managements}

Phosphor $(\mathrm{P})$ ist lebenswichtig für Pflanzen, Tiere und Menschen. Doch obwohl das nicht-substituierbare Nährelement

Dr. agr. Beatrice Garske, M.Sc., LL.M.,

Forschungsstelle Nachhaltigkeit und Klimapolitik,

Leipzig, Deutschland

Prof. Dr. Dr. Felix Ekardt, M. A., LL.M.,

Forschungsstelle Nachhaltigkeit und Klimapolitik,

Leipzig, Deutschland unsere Nahrungsmittelproduktion sichert, ist die P-Düngung in der deutschen und europäischen Landwirtschaft häufig ineffizient. ${ }^{1}$ Während die Böden in einigen Regionen Europas Nährstoffdefizite aufweisen und weniger ertragsfähig werden, werden andernorts, insbesondere in viehreichen Regionen, große Mengen $\mathrm{P}$ im Boden akkumuliert. Dort steigt die Gefahr des P-Austrags und der Gewässereutrophierung. ${ }^{2}$ Die rohphosphathaltigen Düngemittel weisen zudem häufig hohe Belastungen an Cadmium (Cd) und Uran (U) auf und bergen damit eine weitere Gefahr für die Ökosysteme und die menschliche Gesundheit. ${ }^{3}$ Nicht zuletzt ist die EU einschließlich Deutschlands nahezu vollständig auf den Import von Rohphosphat bzw. rohphosphathaltigen Düngemitteln angewiesen. Die endlichen Rohphosphatvorkommen konzentrieren sich auf einige wenige, häufig politisch instabile Weltregionen wie etwa Marokko und die Westsahara, was die Gefahr von Versorgungsengpässen birgt, ${ }^{4}$ weshalb die EU Phosphatgestein und Phosphor als kritische Rohstoffe eingestuft hat. ${ }^{5}$ Statt $\mathrm{P}$

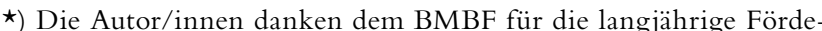
rung des BonaRes-Projektes InnoSoilPhos (No. 031B0509) sowie dem Leibniz WissenschaftsCampus Phosphorforschung Rostock.

1) Schoumans/Bouraoui/Kabbe/Oenema/van Dijk, AMBIO 2015, $180 \mathrm{ff.;}$ Leinweber u. a., AMBIO 2018, $3 \mathrm{ff}$.

2) Sharpley u.a., AMBIO 2015, $163 \mathrm{ff} . ;$ Tóth/Guicharnaud/Tóth/Hermann, Eur J Agron 2014, 42, 49 ff.; HELCOM, Pollution Load Compilation, 2018, S. 22-27.

3) Ulrich, Sci Total Environ 2020, $541 \mathrm{ff}$.; Cordell/Rosemarin/Schröder/ Smit, Chemosphere 2011, 747 ff.; Kratz/Schick/Schnug, Sci Total Environ 2016, $1013 \mathrm{ff}$

4) Cordell/Drangert/White, Glob Environ Change 2009, $292 \mathrm{ff}$.; Rosemarin/Ekane, Nutr Cycl Agroecosyst 2016, 265, 268 f.; Schoumans/ Bouraoui/Kabbe/Oenema/van Dijk, AMBIO 2015, 180.

5) European Commission, COM(2014) 297 final, 26. 5.2014; European Commission, COM(2017) 490 final, 13.9.2017; European Commission, COM(2020) 474 final, 3.9.2020. 
weitestgehend im Kreislauf zu führen, entstehen in der EU ebenso wie in Deutschland jedoch P-Verluste entlang der gesamten Wertschöpfungskette und erhebliche Mengen P in Abwasser- und Abfallströmen bleiben für die Rückgewinnung ungenutzt. ${ }^{6}$ Ein besseres P-Management ist nötig, um die P-Versorgung der europäischen Landwirtschaft sicherzustellen, den Erhalt der Bodenfruchtbarkeit und der Bodenfunktionen langfristig zu gewährleisten und gleichzeitig Umweltschäden wie die Eutrophierung von Gewässern zu vermeiden, P-Kreisläufe zu schließen sowie die P-Rückgewinnung $z u$ fördern und die mineralischen P-Reserven zu schonen. ${ }^{7}$ Dazu gehört es insbesondere, bedarfsgerecht, standort- und kulturspezifisch zu düngen und dadurch PÜberschüsse zu vermeiden. ${ }^{8}$ Ein nachhaltiges P-Management ist zudem in ein nachhaltiges Boden-Management ${ }^{9}$ - als integrativen Bestandteil einer nachhaltigen Landwirtschaft ${ }^{10}$ - eingebettet: Die Reduktion von Bodenerosion verringert auch P-Austräge in die Gewässer. ${ }^{11}$ Ein gelockerter Boden, der reich an Mikroorganismen ist, trägt zur Mobilisierung vorhandener P-Gehalte bei, wofür unter anderem auch diversifizierte Fruchtfolgen vorteilhaft sind. Die mikrobielle Aktivität des Bodens kann außerdem durch verschiedene P-Aktivatoren und durch die Zufuhr organischer Substanz im Rahmen der organischen Düngung (einschließlich Kompost- und Gründüngung) erhöht werden. ${ }^{12}$ Ferner sollten bergbaulich gewonnene P-Dünger zunehmend durch kreislaufwirtschaftsfreundliche organische Dünger und PRezyklate ersetzt werden. ${ }^{13}$

Einen wesentlichen Einfluss auf die P-Bilanz auf betrieblicher, regionaler und europäischer Ebene hat die hohe und weiter steigende Produktion tierischer Nahrungsmittel (sel-

6) Cordell/Neset/Prior, Curr Opin Biotechnol 2012, 839; Van Dijk/ Lesschen/Oenema, Sci Total Environ 2016, 1078, 1082; Jedelhauser/Binder, Res Conserv Rec 2015, 294, 300; Mew/Steiner/Geissler, Sustainability 2018, 1, 4.

7) Die Faktoren eines nachhaltigen P-Managements wurden detailreicher in Garske/Douhaire/Ekardt, NuR 2018, 73 ff. und Garske/ Stubenrauch/Ekardt, RECIEL 2020, $107 \mathrm{ff}$. vorgestellt. Eine ausführliche Herleitung findet sich in Garske, Ordnungsrechtliche und ökonomische Instrumente der Phosphor-Governance. Unter Berücksichtigung der Wirkungen auf Böden, Gewässer, Biodiversität und Klima, 2020.

8) Withers u. a., Environ Res Lett 2017, $1 \mathrm{ff}$.; Tucher/Hörndl/Schmidhalter, AMBIO 2018, $41 \mathrm{ff}$;; Buczko u. a., AMBIO 2018, $50 \mathrm{ff}$.

9) FAO, Voluntary Guidelines for Sustainable Soil Management, 2016.

10) IAASTD, Global Report, 2009, S. $377 \mathrm{ff}$.; Garske, Instrumente (Fn. 7), S. 118 ff.; Stubenrauch, Phosphor-Governance in ländervergleichender Perspektive - Deutschland, Costa Rica, Nicaragua. Ein Beitrag zur Nachhaltigkeits- und Bodenschutzpolitik, 2019, S. $81 \mathrm{ff}$.

11) Withers u. a., Environ Res Lett 2017, 1,12f.; Zimmer/Kahle/Baum: Agric Wat Manage 2016, $21 \mathrm{ff}$.

12) Grafe u. a., Environ Microbiol Reports 2018, $320 \mathrm{ff.;}$ Eichler-Löbermann u. a., Management Options for an Efficient Utilization of Phosphorus in Agroecosystems, in: Schnug/De Kok (Ed): Phosphorus in Agriculture: $100 \%$ Zero, 2016, 179, 185; Eichler-Löbermann/Köhne/Kowalski/Schnug, J Plant Nutr 2008, 659, $670 \mathrm{ff.;}$ Zhu/Li/Whelan, Sci Total Environ 2018, 522,529; Bergkemper u. a., Environ Microbiol 2016, 1988; Peine u. a., J Plant Nutr Soil Sci 2019, 157, $170 \mathrm{ff}$.

13) Roy, Ecol Eng 2017, 213 ff.; Morshedizad/Leinweber, Clean - Soil, Air, Water 2017, 1; Macintosh u. a., Sci Total Environ 2019, 90, 95; Schoumans/Bouraoui/Kabbe/Oenema/van Dijk, AMBIO 2015, 180,184; Van Zanten/Van Ittersum/De Boer, Global Food Secur 2019 18, 21.

14) Svanbäck u.a., Sci Total Environ 2019, 1549 ff.; Bouwman u.a., Proc Natl Acad Sci 2013, 20822, 20885; Smil, Annu Rev Energy Environ 2000, 53, 55; Leip u.a., Environ Res Letters 2015, 1, $6 \mathrm{ff}$. Siehe auch Gethke-Albinus, Verfahren zur Gewinnung von Sekundärrohstoffen aus flüssigen Stoffströmen und deren Einfluss auf die deutsche Phosphorbilanz 2012, S. 127 ff. für eine nationale P-Bilanz, die P-Düngemittel (zum großen Teil auch für den Futtermittelanbau) und die P-Importe für die Futtermittel biges gilt für Stickstoff, N) ${ }^{14}$ Es ist insbesondere die räumliche Trennung von Viehhaltung und Ackerbau, durch welche der P-Kreislauf gestört wird: (Mineralische) P-Dünger müssen in Regionen gebracht werden, in denen Futtermittel angebaut werden. Von diesen Flächen wird P mit der Ernte wieder abtransportiert und in die Regionen mit Tierhaltung gebracht, wo P aus den Futtermitteln nur teilweise von den Tieren verwertet werden kann und der Rest wieder ausgeschieden wird. Die P-haltigen Wirtschaftsdünger wiederum verbleiben häufig in der Region der Tierhaltung und führen dort zu überhöhten Boden-P-Gehalten. ${ }^{15}$ Die (in der Summe und auch regional) hohen Tierzahlen stehen dabei nicht nur der Schließung der P-Nährstoffkreisläufe entgegen, ${ }^{16}$ sondern auch dem Klima- und Biodiversitätsschutz: Bereits heute werden rund $70 \%$ der Weltagrarfläche inklusive der Weideflächen für die Produktion tierischer Nahrungsmittel beansprucht (es werden auch höhere Zahlen genannt). ${ }^{17}$ Die FAO geht bei gleichbleibenden Konsumtrends davon aus, dass der Anteil des verfütterten Getreides von $30 \%$ auf $50 \%$ im Jahr 2050 steigen und entsprechende - klima- und biodiversitätsbeeinträchtigende ${ }^{18}$ - Landnutzungsänderungen mit sich bringen wird. ${ }^{19}$ Gleichzeitig ist die Tierhaltung wesentlicher Emittent von Treibhausgasen. Es entsteht Kohlenstoffdioxid $\left(\mathrm{CO}_{2}\right)$ durch Humusabbau, etwa in Folge von Landnutzungsänderungen, Methan $\left(\mathrm{CH}_{4}\right)$ aus den Verdauungsprozessen der Nutztierhaltung sowie $\mathrm{CH}_{4}$ und Lachgas $\left(\mathrm{N}_{2} \mathrm{O}\right)$ durch die Wirtschaftsdüngerausbringung und -lagerung und aus den Böden infolge der N-Düngung. ${ }^{20}$.

Nicht zuletzt entstehen in der Tierhaltung sowie in der gesamten Landwirtschaft Treibhausgase durch die Nutzung fossiler Brennstoffe, etwa für den Transport, die Bo-

als größte P-Importquellen nach Deutschland identifiziert; ferner Jedelhauser/Binder, Res Conserv Rec 2015, 294, 300 für eine europäische P-Bilanz mit übereinstimmenden Befunden.

15) Tóth/Guicharnaud/Tóth/Hermann, Eur J Agron 2014, 46 i. V.m. Eurostat, Livestock density by NUTS2 regions, EU28, 2016, Stand 30.7.2020, abrufbar unter https://ec.europa.eu/eurostat/statistics-explained/index.php?title=File:Map1_Livestock density_NUTS2_EU-28_2016.png; Einarsson/Pitulia/Cederberg, Nutr Cycl Agroecosyst 2020, 199, 206; Sharpley u.a., AMBIO 2015, 163, 166 und 171; Bomans u. a., Adressing phosphorus related problems in farm practice. Final report of the Soil Service of Belgium to the European Commission, 2005, S. 15 und S. $40 \mathrm{ff}$.; Svanbäck u. a., Sci Total Environ 2019, $1549 \mathrm{ff}$.

16) Bouwman u.a., Proc Natl Acad Sci 2013, 20822; Metson/Bennett/ Elser, Environ Res Lett 2012, 1 ff.; Prud'homme/Heffer, Fertilizer Outlook 2017-2021, 2017; Kebreab, Sustainable Animal Agriculture, 2013, S. $127 \mathrm{ff}$.

17) FAO, Livestock's Long Shadow. Environmental Issues and Options, 2006, S. xxi; UNEP, Global Environment Outlook 6, 2019, S. 202; Mottet u. a., Food Secur Gov Lat Amer 2017, 1, 5

18) IPCC, Climate Change and Land: Summary for policymakers, 2020, S. $7 \mathrm{ff}$; IPCC, Climate Change 2014: Synthesis Report, 2014, S. 384 und S.822ff.; Secretariat of the CBD, Global Biodiversity Outlook 4, 2014, S.25; IPBES, The global assessment report on biodiversity and ecosystem services: Summary for policymakers, 2019, S. $12 \mathrm{ff}$.; IAASTD, Report (Fn. 10), S. $11 \mathrm{ff}$; Millennium Ecosystem Assessment, Ecosystems and Human WellBeing: Synthesis, 2005.

19) Phelps/Kaplan, Glob Chang Biol 2017, $4457 \mathrm{ff}$; Berners-Lee/ Kennelly/Watson/Hewitt, Elem Sci Anth 2018, 1, 10; Smith u.a., Glob Chang Biol 2013, 2285 ff.; FAO, Livestock's long shadow, 2006, S. 12 sowie das FAO-Szenario FAO, How to Feed the World in 2050, 2009 und seine Neuauflage FAO, World Agriculture towards 2030/2050, 2012. Zur Kritik der wachstumsbasierten FAO-Szenarien, Smil, Glob Food Sec 2014, 67, 68. Ferner auch Bodirsky u.a., PLOS 2015, $1 \mathrm{ff}$. und Springmann u. a., Nature 2018, 519 ff. sowie Bouwman u. a., Proc Natl Acad Sci 2013, $20882 \mathrm{ff}$.

20) IAASTD, Report (Fn. 10), S. 160; IPCC, Climate Change 2014 (Fn. 18), S. 840; FAO, Agriculture, Forestry and Other Land Use Emissions by Sources and Removals by Sinks, 2014, S. 8 ff.; Gerber/Portet/Key/Steinfeld, Anim Int J Anim Biosci 2010, 393, 394; Jackson u. a., Environ Res Let 2020, 1. 
denbearbeitung und vor allem durch die energieintensive Herstellung von N-Düngern im Haber-Bosch-Verfahren. ${ }^{21}$ Zumindest ein Teil dieser Emissionen aus den Bereichen Tierhaltung, Düngung und Böden gilt als unvermeidbar, wenn weiterhin Landwirtschaft betrieben wird. Das gilt selbst bei veganer Ernährung, aber erst recht, wenn zumindest ein Teil der (Weide-)Tierhaltung weiter praktiziert wird, was für die Welternährungslage und teils für die Biodiversität auch Vorteile bietet. ${ }^{22}$ Dies erhöht den Druck auf Verminderungsmaßnahmen in anderen Bereichen sowie auf Kompensationsmaßnahmen wie Aufforstungen, Kohlenstoffspeicherung der Böden, Wiedervernässung von Feuchtgebieten wie Mooren u. ä. ${ }^{23}$

Es bedarf insofern integrierter Lösungen für die P-bezogenen Problemlagen und die damit verknüpften landwirtschaftlichen Probleme. Strategisch dürfte es nach alledem naheliegen, die landwirtschaftliche P-Nutzung so $\mathrm{zu}$ gestalten, dass die P-Effizienz im Sinne einer effizienten, bedarfsbasierten, standort- und kulturspezifischen P-Düngung erhöht wird, insbesondere unter Berücksichtigung der Boden-P-Gehalte sowie unter Anwendung Pmobilisierender Maßnahmen. Auch die Effizienz in der Tierfütterung sollte gesteigert werden. P-Überschüsse sollten weitestgehend ausgeschlossen werden, so dass das Eutrophierungsrisiko vermindert wird. Zudem sollten Schadstoffbelastungen mit $\mathrm{Cd}$ und $\mathrm{U}$ eliminiert werden. Die Importabhängigkeit von $\mathrm{P}$ (auch bezogen auf Futtermittel) sollte verringert und die P-Kreislaufwirtschaft im Sinne der Substitution rohphosphathaltiger Düngemittel durch organische Dünger und rückgewonnenen $\mathrm{P}$ gefördert werden. Gleichzeitig sollten der Ökolandbau und andere agrarökologische Anbausysteme begünstigt und Viehhaltung und Ackerbau im Sinne geschlossener P-Kreisläufe wieder zusammengeführt werden. ${ }^{24}$ Terminologisch würde dies im Sinne der gängigen Nachhaltigkeitsstrategien Aspekte von Konsistenz, Effizienz und Suffizienz kombinieren. ${ }^{25}$

Der vorliegende Artikel konzipiert Kernelemente einer P-Governance so, dass sie neben $\mathrm{P}$ insbesondere auch die klima- und biodiversitätsbezogenen Herausforderungen der Landwirtschaft mit einbezieht und die zu entwickelnden Instrumente daran ausrichtet, dass sie zur Erreichung der international verbindlichen Umweltziele beitragen, das heißt zur Begrenzung der Erderwärmung auf deutlich unter $2^{\circ} \mathrm{C}$ und möglichst $1,5^{\circ} \mathrm{C}$ gemäß Art. 2 Abs. 1 Paris-Abkommens (PA) und zum Stopp des Biodiversitätsverlustes gemäß den Aichi-Zielen B und $\mathrm{C}$ zur Biodiversitätskonventionen (CBD). Die Abkommen implizieren Nullemissionen und damit einen Ausstieg aus den fossilen Brennstoffen in höchstens zwei Dekaden sowie eine starke Verringerung der Tieranzahl. ${ }^{26}$ Sie machen also umfassende Veränderungen in allen Lebensbereichen einschließlich des Agrarsektors notwendig und haben damit auch erhebliche P-Auswirkungen.

Was genau die angemessene Nachhaltigkeitsstrategie ist (für P wie auch generell für die Landwirtschaft), lässt sich allerdings erst sagen, wenn die zu verfolgenden Ziele feststehen und gegebenenfalls untereinander abgewogen wurden (dazu siehe auch Kapitel 3.3.2). Denn bislang fehlt es an einer übergreifenden verbindlichen Zielstellung direkt für P. Dies hat neben offenen normativen Fragen des nachhaltigen Umgangs mit endlichen Ressourcen auch mit offenen empirischen Fragen sowie einer großen empirischen Heterogenität zu tun, etwa hinsichtlich der gegenwärtigen Boden-P-Gehalte und der Frage danach, von welcher Art der Ernährung man künftig ausgeht (und deren P-Intensität stark variiert). Insoweit besteht sowohl politisch als auch wissenschaftlich weiterer Klärungsbedarf, auch wenn ein sparsamer Umgang mit der endlichen Ressource P ebenso wie ein besserer Umwelt- sowie Gesundheitsschutz und damit die oben genannten Strategien tentativ naheliegen - zumal sie unmittelbar mit einer geringeren Tierhaltung verknüpft sind, die ihrerseits bereits durch die Klima- und Biodiversitätsziele eingefordert wird.

Orientiert an den oben genannten Zielstellungen zur PNutzung wird im Folgenden im Anschluss an eine frühere Publikation $^{27}$ zunächst zusammengefasst und auf den aktuellen Stand gebracht, welche Rechtsbereiche des deutschen und europäischen Agrar- und Umweltrechts für die P-Düngung relevant sind - und welche Defizite der auf Details und Einzelvorgänge bezogene, zumeist ordnungsrechtlich geprägte Rechtsrahmen generell aufweist. Darauf aufbauend werden die Vorteile ökonomischer Instrumente bei der Lösung von mengenbezogenen Nachhaltigkeitsproblemen vorgestellt. Sodann werden optimierende ökonomische Instrumente für die Landwirtschaft diskutiert, wobei ein Primärenergie-Emissionshandel und ein Emissionshandel für tierische Produkte in den Fokus rücken. Es wird geprüft, inwieweit durch eine derartige Adressierung von Klima und Biodiversität auch $\mathrm{P}$-spezifische Probleme gelöst werden und welcher Ergänzungsbedarf durch P-bezogene Instrumente noch besteht. Methodisch erfolgt dies im Sinne einer qualitativen Governance- oder Rechtswirkungsanalyse. Diese bestimmt speziell bei hypothetischen also noch nicht real umgesetzten - Rechtsinstrumenten deren Wirksamkeit gemessen an den jeweiligen Zielen unter Berücksichtigung typischer Steuerungsprobleme (dazu das nächste Kapitel), deren Existenz aus empirischen Beobachtungen und aus interdisziplinären verhaltenswissenschaftlichen Erkenntnissen abgeleitet werden kann. ${ }^{28}$

\section{Defizite des Ordnungsrechts und Lösungsoptionen durch ökonomische Instrumente}

Die einzelnen Aspekte des nachhaltigen P-Managements berühren verschiedene Rechtsbereiche der EU bzw. der Nationalstaaten. Neben der Gemeinsamen Agrarpolitik der EU (GAP) erweisen sich das Bodenschutzrecht, das Gewässerschutzrecht, die anwendungs- und produktbezogenen Vorschriften des Dünge(mittel)rechts sowie mit Blick auf das P-Recycling auch das Kreislaufwirtschaftsrecht als relevant. Bezogen auf den Betrieb von Tierhaltungsanlagen

21) IPCC, Climate Change 2014 (Fn. 18), S. 822 ff.; Kyriakou/Garagounis/Vourros/Vasileiou/Stoukides, Joule 2020, $142 \mathrm{ff.;}$ Razon, Environ Prog Sustainable Energy 2018, 618 ff.; Sutton u. a., Our Nutrient World. The Challenge to Produce More Food and Energy with Less Pollution, 2013, S. 8 und S. 16; Weishaupt/Ekardt/ Garske/Stubenrauch/Wieding, Sustainability 2020, 1, 5.

22) Stoll-Kleemann/Schmidt, Glob Environ Change 2017, 1261, 1262 Rook/Tallowin, Anim Res 2003, $181 \mathrm{ff}$.

23) Mengis/Matthews, npj Clim Atmos Sci 2020, $1 \mathrm{ff.;}$ Ekardt/Wieding/ Zorn, Sustainability 2018, $1 \mathrm{ff}$;; Ekardt/Jacobs/Stubenrauch/Garske, Land 2020, 1 ff.; Lóránt/Allen, Net-zero agriculture in 2050: how to get there? Report by the Institute for European Environmental Policy, 2019, S. 6 ff.

24) Ausführlich Garske, Instrumente (Fn. 7), Kapitel 2.2 und Kapitel 5 .

25) Ausführlich dazu Ekardt, Sustainability: Transformation, Governance, Ethics, Law, 2019, Chapter 1.3; Stubenrauch, PhosphorGovernance, Kapitel 4.2 (Fn. 10).

26) Ekardt, Sustainability (Fn. 25); Garske, Instrumente (Fn. 7); Stubenrauch, Phosphor-Governance (Fn. 10); Weishaupt, Nachhaltigkeits-Governance tierischer Nahrungsmittel in der EU, 2019; IPCC, Global Warming of $1,5^{\circ} \mathrm{C}, 2018$; Rockström u. a., Science 2017, 1269; Steffen u.a., PNAS 2018, $8252 \mathrm{ff.;}$ Ekardt/Wieding/ Zorn, Sustainability 2018, $1 \mathrm{ff}$.; Mengis/Matthews, npj Clim Atmos Sci 2020, $1 \mathrm{ff}$;; European Commission, COM(2020) 381 final, 20.5.2020; European Commission, $\operatorname{COM}(2019) 640$ final, 11. 12.2019; IPBES, Assessment (Fn. 18), S. 39 ff.; IAASTD, Report (Fn. 10), S. $379 \mathrm{ff}$

27) Garske/Douhaire/Ekardt, NuR 2018, 73 ff.

28) Weishaupt/Ekardt/Garske/Stubenrauch/Wieding, Sustainability 2020 1, 2-4; Garske/Heyl/Ekardt/Weber/Gradzka, Land 2020, 1, 2-4; Ekardt/Jacobs/Stubenrauch/Garske, Land 2020, 1, 2-3; Stubenrauch/ Ekardt, Environments 2020, 1, 2-3. 
sind zudem das Bau- und Planungsrecht sowie das immissionsschutzrechtliche Genehmigungsrecht von Bedeutung. Ebenfalls bedeutend erweist sich das Recht des Ökolandbaus, welches einige Regulierungsimpulse für ein nachhaltigeres P-Management enthält. Eine ausführliche Analyse dieser Rechtsbereiche ergab, dass an verschiedenen Stellen zwar einige wichtige Ansatzpunkte für ein nachhaltigeres P-Management verankert sind, eine ausreichende Steuerungswirkung jedoch ausbleibt. ${ }^{29}$ Insbesondere die für die P-Problematik relevante Tierdichte und hohe Tieranzahl, welche die klima- und biodiversitätspolitischen Ziele im besonderen Maße konterkariert, wird durch keine der bestehenden Regelungen ausreichend adressiert. Eine Ausnahme bildet hier lediglich der Ökolandbau, der in Art. 5 lit. g) und Art. 14 (1) lit. d) Nr. i) VO (EG) 834/200730 eine Kopplung der Tieranzahl an die Fläche unter Berücksichtigung des Futtermittelbedarfs und der Menge der anfallenden Wirtschaftsdünger vorsieht, wenngleich auch dort begriffliche Unschärfen und dadurch Schlupflöcher, etwa bezüglich der „Region“ des Futtermittelanbaus, bestehen. ${ }^{31}$ Allerdings werden bislang nur 7,5\% der landwirtschaftlichen Nutzfläche der EU ökologisch bewirtschaftet, ${ }^{32}$ und letztlich bleibt der Ökolandbau auch dann ein Randphänomen, wenn das Ziel der im Mai 2020 von der EU-Kommission veröffentlichten Farm-to-Fork-Strategie erreicht werden sollte, den Ökolandbau bis 2030 auf $25 \%$ der Agrarfläche auszuweiten. ${ }^{33}$ Unabhängig davon bleibt auch im Ökolandbau eine Begrenzung der Tierzahlen entsprechend dem Klimaziel aus. Nichtsdestotrotz enthält der Okolandbau neben der Flächenbindung auch weitere Ansatzpunkte für ein nachhaltiges P-Management, insbesondere den Erhalt der natürlichen Bodenfruchtbarkeit und des Bodenlebens sowie die Beschränkung außerbetrieblicher Produktionsmittel einschließlich leichtlöslicher mineralische Düngemittel (Art. 5 lit. a), b), Art. 4 lit. b) Nr. iii) VO (EG) 834/2007), die Vermeidung von Bodenerosion sowie den Erhalt der organischen Bodensubstanz (Art. 12 (1) lit. a) VO (EG) 834/2007) und ist insofern auch aus PPerspektive förderwürdig. Dennoch gilt es, auch auf ökologisch bewirtschafteten Flächen P-Überschüsse zu vermeiden, aber auch P-Defizite, wie sie häufig bei viehlosen Ökolandbaubetrieben auftreten. ${ }^{34}$

Abgesehen von den seit langem aufgrund ihrer unzureichenden Umweltwirkung in der Kritik stehenden Subventionsregelungen im Rahmen der europäischen GAP $^{35}$ ist der gegenwärtige Rechtsrahmen stark ordnungsrechtlich geprägt und beinhaltet detaillierte Regelungen für Einzelvorgänge und -produkte. Die vor allem für das Ordnungsrecht charakteristische Orientierung an einzelnen Anlagen, Produkten oder Tätigkeiten weist jedoch Defizite bei der Lösung mengenbezogener Nachhaltigkeitsprobleme auf. Zunächst ist es gerade im Umweltbereich und speziell auch für P und Böden schwierig, komplexe Ursache-WirkungBeziehungen wie jene zwischen Ökosystemen und den teils multikausalen Umweltschäden zu erfassen und in detaillierten ordnungsrechtlichen Regeln abzubilden (Abbildbarkeitsprobleme). Zudem kompensieren Rebound-Effekte regelmäßig die Effizienzgewinne in Einzelbereichen. Sektorale, räumliche oder von Umweltproblem zu Umweltproblem wirkende Verlagerungseffekte führen außerdem regelmäßig dazu, dass Probleme schlicht von einem regulierten in einen weniger regulierten Bereich verschoben werden. Ferner sind - was in der Vielzahl ordnungsrechtlicher Normierungen meist untergeht - die materiellen Vorgaben der Normen oftmals nicht ambitioniert genug, um die verfolgten übergreifenden Ziele effektiv umzusetzen. Gleichzeitig beeinträchtigen Ausnahmetatbestände und Schlupflöcher die Wirksamkeit der rechtlichen Vorgaben (mangelnde Zielstrenge). Hinzu kommen regelmäßig Vollzugsprobleme, gerade für schwer zu fassende und kaum überwachbare diffuse Quellen wie die Landwirtschaft mit ihrer Vielzahl einzelner Vorgänge. ${ }^{36}$
Direkt oder indirekt mengensteuernd wirkende Instrumente, das heißt ökonomische Instrumente wie Abgaben oder Cap-and-Trade-Systeme (Emissions- bzw. Zertifikathandelssysteme), ${ }^{37}$ haben gegenüber ordnungsrechtlichen (Detail-)Regelungen bei Nachhaltigkeitsfragen das Potenzial, die aufgelisteten Steuerungsprobleme zu vermeiden, sofern man bestimmte Ausgestaltungen wählt. Knüpft man das Mengen-Cap oder den Preis an eine leicht fassbare Steuerungsgröße (wie die fossilen Brennstoffe) und wendet das System sektoral und räumlich breit an, sind Vollzugs- und Abbildbarkeitsprobleme, sektorale und räumliche Verlagerungseffekte ganz oder weitgehend ausgeschlossen (spätestens durch ergänzende Border Adjustments). Zudem werden aufgrund der absoluten Mengengrenze bei Caps (nicht dagegen bei Abgaben) Rebound-Effekte ausgeschlossen. Okonomische Instrumente sind dabei neutral gegenüber den verschiedenen Nachhaltigkeitsstrategien, denn sie überlassen den Normadressaten, bei denen der explizite oder über die Mengenverknappung erzeugte implizite Preisdruck ankommt, ob sie auf Konsistenz, Effizienz oder Suffizienz setzen. Dies zeigt im Sinne liberal-demokratischer Grundprinzipien zugleich, dass solche Steuerungsansätze nicht nur ökologisch effektiv im Sinne von zielerreichend und steuerungsproblemvermeidend sind, wenn man sie in der beschriebenen Weise ausgestaltet, sondern dass sie aufgrund der ihnen belassenen Flexibilität auch mehr Freiheitsfreundlichkeit bei gleichzeitiger Verursacherorientierung und ökonomischer Kostengünstigkeit für die Normadressaten versprechen. Ebenso wie für ordnungsrechtliche (Detail-)Regelungen gilt jedoch auch für ökonomische Instrumente, dass sie dem verfolgten Ziel entsprechend streng sein müssen - dass also die Mengenbegrenzung entspre-

29) Garske/Douhaire/Ekardt, NuR 2018, 73, 75 ff.; Garske, Instrumente (Fn. 7); Stubenrauch, Phosphor-Governance (Fn. 10); Douhaire, Rechtsfragen der Düngung. Eine steuerungs- und rechtswissenschaftliche Analyse vor dem Hintergrund unions- und völkerrechtlicher Verpflichtungen und politischer Zielsetzungen zum Umwelt- und Ressourcenschutz, 2019.

30) Verordnung (EG) Nr. 834/2007 des Rates vom 28.6.2007 über die ökologische/biologische Produktion und die Kennzeichnung von ökologischen/biologischen Erzeugnissen und zur Aufhebung der Verordnung (EWG) Nr. 2092/91, ABl. L 189, S. 1.

31) $\mathrm{Ab}$ 1.1.2021 wird die Verordnung (EG) Nr. 834/2007 durch die Verordnung (EU) 2018/848 des Europäischen Parlaments und des Rates vom 30.5.2018 über die ökologische/biologische Produktion und die Kennzeichnung von ökologischen/biologischen Erzeugnissen sowie zur Aufhebung der Verordnung (EG) Nr. 834/2007 des Rates, ABl. L 150, S. 1 ersetzt.

32) Eurostat, Organic crop area by agricultural production methods and crops (from 2012 onwards), Stand 27. 7.2020, abrufbar unter https://appsso.eurostat.ec.europa.eu/nui/submitViewTable Action.do.

33) European Commission, COM(2020) 381 final, 20.5.2020, S. 9.

34) Ohm/Paulsen/Moos/Eichler-Löbermann, Agron Sustainable Dev 2017, $1 \mathrm{ff}$

35) Pe'er u.a., Is the CAP Fit for purpose? An evidence-based fitness-check assessment, 2017; Garske/Hoffmann, Die Gemeinsame Agrarpolitik nach der Reform 2013: Endlich nachhaltig? 2016. Für die aktuellen Verordnungsvorschläge siehe European Commission, COM(2018) 392 final, 393 final und 394 final, 1.6.2020. Für eine Kritik dieser Vorschläge siehe Heyl/Döring/Garske/Stubenrauch/Ekardt, RECIEL 2020, $1 \mathrm{ff}$.; Pe'er u. a., People and Nature 2020, $305 \mathrm{ff}$

36) Ekardt, Sustainability (Fn. 25), Chapter 4.4; Weishaupt/Ekardt/ Garske/Stubenrauch/Wieding, Sustainability 2020, 1, 2f.; Garske/ Heyl/Ekardt/Weber/Gradzka, Land 2020, 1, 3 f.

37) Subventionen werden als Sonderfall ökonomischer Instrumente nicht näher betrachtet. Subventionen spielen insbesondere im Rahmen der GAP eine große Rolle für den Landwirtschaftssektor. Kritisch dazu Heyl/Döring/Garske/Stubenrauch/Ekardt, RECIEL 2020, $1 \mathrm{ff}$. und Pe'er u. a., People and Nature 2020, $305 \mathrm{ff}$ Ferner $U B A$, Umweltschädliche Subventionen in Deutschland, 2016, S. $57 \mathrm{ff}$ 
chend ambitioniert bzw. der Steuersatz ausreichend hoch sein muss. ${ }^{38}$

Die Begrifflichkeit ,ökonomische Instrumente versus Ordnungsrecht" ist bei alledem durchaus anfällig für Missverständnisse. ${ }^{39}$ Der Hauptunterschied bei den Governance-Instrumenten ist weniger der zwischen „Preis“ und „Verbot“ als jener zwischen Mengen- und Detailsteuerung. Dies ist bereits terminologisch richtig, denn ein Cap null könnte auch als Verbot bezeichnet werden. Demgegenüber haben Verbote auch Preiswirkungen. Beispielsweise können ordnungsrechtliche Anforderungen an Schadstoffgehalte die Preise für rohphosphathaltige Düngemittel erhöhen und damit die Verwendung von recycelten P-Düngern und den Einsatz organischer Düngemittel fördern. Wie immer man es fasst - im Düngerecht finden ökonomische respektive mengenorientierte Instrumente bislang kaum Anwendung. In Deutschland gerieten sie in den letzten Jahren im Zuge des Vertragsverletzungsverfahrens ${ }^{40}$ aufgrund des Verstoßes gegen die EU-Nitratrichtlinie ${ }^{41}$ in die politische und wissenschaftliche Diskussion, allerdings mit klarem Fokus auf N. ${ }^{42}$ Daher soll im Folgenden geprüft werden, ob ökonomische Instrumente auch für die mit der P-Nutzung verbundenen Probleme effektive Lösungen bieten können.

\section{3. Ökonomische Instrumente für die P-Governance und verknüpfte Umweltprobleme}

Näher analysiert werden im Folgenden zunächst zwei mögliche Steuerungsinstrumente: ein auf alle fossilen Brennstoffe ausgedehnter reformierter EU-Emissionshandel der ersten Handelsstufe, durch den die fossilen Brennstoffe in maximal zwei Dekaden aus dem Markt genommen wer$\operatorname{den}^{43}$, und ein separater Emissionshandel für tierische Produkte, der die Anzahl der Tiere entsprechend dem Klimaund Biodiversitätsziel stark reduziert. ${ }^{44}$ Bezogen auf $\mathrm{P}$ stellt sich sodann die Frage, inwieweit jene übergreifenden Nachhaltigkeitsinstrumente bereits auf die erläuterten $\mathrm{P}$ bezogenen Problemlagen bzw. die oben formulierten Zielsetzungen wirken und welcher weitere Steuerungsbedarf diesbezüglich bestehen bleibt.

\subsection{Primärenergie-Emissionshandel}

Nicht nur die landwirtschaftliche Erzeugung, sondern ein Großteil der Wirtschaft in den Industrieländern basiert auf der Nutzung fossiler Brennstoffe, die seit der Industrialisierung zudem eine wesentlichen Wohlstandsquelle waren. Sie werden als Treibstoffe in Verkehr und Industrie, für Strom und Wärme, für die Herstellung von Düngemitteln, Chemikalien, Kunststoffen, Pharmazeutika etc. genutzt. Die fossilen Brennstoffe durchziehen nahezu alle Lebensbereiche. Dementsprechend bieten sie einen sektoral breiten und zugleich leicht fassbaren Ansatzpunkt für eine integrierte Lösung verschiedener Umweltprobleme. ${ }^{45}$

Im Idealfall würde ein globales und alle Sektoren umfassendes Primärenergie-Emissionshandelssystem den Einsatz fossiler Brennstoffe in höchstens zwei Dekaden durch eine schrittweise abzusenkende, absolute Mengenbegrenzung (Cap) auf null begrenzen. ${ }^{46}$ Solange keine solch globale Lösung umgesetzt werden kann, könnte auch der EUEmissionshandel auf einen primärenergiebasierten Ansatz umgestellt und in puncto Cap am Pariser Klimaziel ausgerichtet werden. Border Adjustments könnten dann Verlagerungseffekte in Länder ohne ähnlichen Ansatz vermeiden. ${ }^{47}$ Dies wird derzeit im Rahmen des Green Deals auf EU-Ebene diskutiert. ${ }^{48}$

Das Ansetzen an der ersten Handelsstufe würde eine weitgehende Abdeckung sämtlicher $\mathrm{CO}_{2}$-Emissionen (und zugleich eine Reduktion einiger anderer THG-Emissionen) gewährleisten. Das Preissignal aus dem ETS kann von den Primärenergieunternehmen über die Strom-, Treibstoff- und Wärmepreise und daran anknüpfend über jede
Art von energieabhängig hergestellten Produkten an alle Unternehmen und Verbraucher weitergegeben werden. Wegen der geringeren Anzahl an Normadressaten würden auch der Vollzug erleichtert und die Kontrollkosten verringert. $^{49}$

Geht man von der Herausnahme fossiler Brennstoffe aus dem Markt (Phasing Out) in maximal zwei Dekaden aus, so stellt dies insbesondere auch den Agrarsektor vor große Herausforderungen. Es ist davon auszugehen, dass bislang häufig energieineffiziente Landmaschinen zukünftig effizienter und mit erneuerbaren Energien betrieben werden können und dass die Digitalisierung und der technische Fortschritt einschließlich Methoden des Precision Farming ein hohes Potenzial zur Steigerung der Effizienz nicht nur der Düngung u. ä., sondern auch bezüglich des Energieeinsatzes bergen. ${ }^{50}$ Dennoch werden die Erzeugung der erneuerbaren Energieträger, das heißt ihr Anbau oder ihre Herstellung, ihr Betrieb und nicht zuletzt auch ihre Entsorgung stets mit einem gewissen Ressourcenverbrauch und Eingriffen in Ökosysteme verbunden sein. ${ }^{51}$ Deshalb, vor allem aber, weil für eine rein technische Strategie die Herausforderung von Nullemissionen in maximal zwei Dekaden wohl zu groß sein wird, würde der reformierte Emissions-

38) Ekardt, Sustainability (Fn. 25), Chapter 4.5; Garske, Instrumente (Fn. 7), Kapitel 4; Schröter-Schlaak/Mewes/Ring, Instrumente des Biodiversitätsschutzes im Politikmix, in: Hansjürgens (Hrsg.): Inwertsetzung biologischer Vielfalt. Naturschutzanliegen in andere Politikbereiche integrieren, 2015, 135,137; Ecotec u.a., Study on the Economic and Environmental Implications of the Use of Environmental Taxes and Charges in the European Union and its Member States. Final Report, 2001, S.i; WHO, Economic Instruments as a lever for policy, 2019.

39) Ekardt/Klinski/Schomerus, Konzept für die Fortentwicklung des deutschen Klimaschutzrechts, 2015.

40) EuGH, Urt. v. 21.6.2018, Rs. C-543/16 - Nitrat; Europäische Kommission, Urt. des Gerichtshofs (Neunte Kammer) v. 21.6.2018 in der Rs. C-543/16, ABl. C 285/6 vom 13.8.2018 - Europäische Kommission/Bundesrepublik Deutschland. Eine Besprechung des Urteils findet sich in Douhaire, ZUR 2018, $464 \mathrm{ff}$.

41) Richtlinie 91/676/EWG des Rates vom 12.12.1991 zum Schutz der Gewässer vor Verunreinigung durch Nitrat aus landwirtschaftlichen Quellen, AB1. L 375, S. 1.

42) SRU, Stickstoff: Lösungsstrategien für ein drängendes Umweltproblem. Sondergutachten, 2015, S. 344, Rdnr. 453 ff.; Möckel, Rechtsgutachten zur Klärung von Rechtsfragen zur Erhebung einer Abgabe auf Stickstoffüberschuss und einer Abgabe auf stickstoffhaltigen Mineraldünger durch den Landesgesetzgeber, 2017, S. $15 \mathrm{ff}$.

43) Ekardt, Sustainability (Fn. 25), Chapter 4.6; Ekardt/Wieding/Zorn, Sustainability 2018, $1 \mathrm{ff}$.

44) Weishaupt/Ekardt/Garske/Stubenrauch/Wieding, Sustainability 2020, 1, $8 \mathrm{ff}$.; ausführlich Weishaupt, Nachhaltigkeits-Governance (Fn. 26)

45) Ekardt, Sustainability (Fn. 25), Chapter 1.2 und passim

46) IPCC, $1,5^{\circ} \mathrm{C}$ (Fn. 26); Ekardt, Sustainability (Fn. 25); Chapter 4; Ekardt/Wieding/Zorn, Sustainability 2018, 1 ff.; Rockström u.a., Science 2017, 1269; Steffen u. a.; PNAS 2018, 8252ff. Zur Alternativoption eines globalen, hohen $\mathrm{CO}_{2}$-Preises siehe insbesondere Nordhaus, A Question of Balance: Weighing the Option on Global Warming Policies, 2008, S. $18 \mathrm{ff}$

47) Ekardt, Sustainability (Fn. 25), Chapter 4.9; Becker/Will, Die Durchsetzbarkeit produktbezogener Border Adjustments, in: Ekardt/Unnerstall/Garske (Hrsg.): Globalisierung, Freihandel und Umweltschutz in Zeiten von TTIP, 2016, $197 \mathrm{ff}$.

48) European Commission, COM(2019) 640 final, 11.12.2019.

49) Ekardt, Sustainability (Fn. 25), Chapter 4; Deutscher Bundestag, WD $8-3000-013 / 18$ v. 28.3.2018, S. 13.

50) Finger/Swinton/Benni/Walter, Annu Rev Resour Econ 2019, 313, 322; Rodias и. a. Sustainability 2017, $1 \mathrm{ff}$.

51) Siehe etwa Hennig, Nachhaltige Landnutzung und Bioenergie. Ambivalenzen, Governance, Rechtsfragen, 2017 zur Bioenergie und European Commission, COM(2018) 562 final, 31. 7.2018, S. 12 zu einigen Problemen der Offshore-Windenergie. 
handel wohl auch Suffizienz auslösen. Sowohl Effizienz, die verstärkte Nutzung erneuerbarer Energien (Konsistenz) als auch Suffizienz werden im Zuge steigender Energiepreise durch die Herausnahme fossiler Brennstoffe aus dem Markt angeregt.

Der Energiepreis gilt außerdem als einer der Einflussfaktoren auf die Preise für Phosphatgestein und P-Düngemittel. ${ }^{52}$ So werden die um das Jahr 2008 um bis zu $800 \%$ gestiegenen Weltmarktpreise für Phosphatgestein in $\mathrm{Zu}-$ sammenhang mit dem Schock des Ölpreises im Jahr 2008 gebracht, selbiges gilt für die Preissteigerungen in den Jahren 1974/75, ${ }^{53}$ wenngleich der Zusammenhang regelmäßig auch als relativ moderat eingeschätzt wird. ${ }^{54}$ Ein Großteil der Rohphosphate wird unter Zugabe von Schwefelsäure $\left(\mathrm{H}_{2} \mathrm{SO}_{4}\right)$ aufgeschlossen, um Phosphorsäure $\left(\mathrm{H}_{3} \mathrm{PO}_{4}\right) \mathrm{zu}$ gewinnen, die Ausgangsstoff verschiedener P-Dünger ist. ${ }^{55}$ Dieser Verfahrensschritt ist eine exotherme Reaktion, bei der Energie entsteht, die gegebenenfalls für den weiteren Verarbeitungsprozess eingesetzt werden kann. Bei modernen Verfahren zur Herstellung von Triple-Superphosphat kann die Gesamt-Energiebilanz sogar positiv ausfallen, ältere Verfahren verbrauchen hingegen Energie. Hinzu kommt der Energieverbrauch für den Düngemitteltransport, der im Falle von P-Düngern aufgrund der langen Transportwege von den Rohstofflagerstätten zu den Produktions- und Verwendungsorten relativ hoch ist. ${ }^{56} \mathrm{Zu}-$ dem entstehen für jede Tonne $\mathrm{P}_{2} \mathrm{O}_{5}$ im Endprodukt durchschnittliche fünf Tonnen Phosphorgips, der größtenteils auf Halden entsorgt werden muss, was ebenfalls einen hohen Transportenergiebedarf bedeutet. ${ }^{57}$ Nicht zuletzt benötigt auch die Düngemittelausbringung Energie, wobei der Aufwand von der Feldgröße, der verwendeten Technik und der Ausbringungsmenge abhängig ist. ${ }^{58}$ Grundsätzlich ist durch die Verteuerung fossiler Brennstoffe demnach auch eine Verteuerung von bergbaulich gewonnenen PDüngern anzunehmen, da Abbau-, Verarbeitungs-, Transport-, und Ausbringungskosten steigen. Im Vergleich zur Produktion von N-Düngemitteln mit Hilfe des HaberBosch-Verfahrens verbraucht die Produktion von P-Düngemitteln jedoch deutlich weniger Energie. Die N-Düngerproduktion durch das Haber-Bosch-Verfahren ist der Faktor mit dem höchsten Energie-Input des Agrarsektors und macht rund 1-2\% der weltweiten Energienachfrage aus. ${ }^{59}$ Durch eine absolute Mengenbegrenzung für fossile Brennstoffe würden synthetische N-Dünger kaum in so hohem Umfang wie heute hergestellt werden können, was einen entscheidenden Einfluss auf die Landwirtschaftspraktiken hätte (insbesondere auch im Zusammenwirken mit der sogleich darzustellenden Regulierung tierischer Produkte). ${ }^{60}$ Grundsätzlich kann der für die Ammoniaksynthese notwendige Wasserstoff sowohl effizienter als auch mit Hilfe erneuerbarer Energien hergestellt werden und auch der (hohe) Energiebedarf der Ammoniaksynthese könnte letztlich durch erneuerbare Energien gedeckt werden. ${ }^{61}$ Die Umsetzung der 2020 veröffentlichten Wasserstoffstrategien Deutschlands ${ }^{62}$ und der EU ${ }^{63}$ läuft jedoch gerade erst an, und derartige Verfahren werden bislang vor allem dort genutzt, wo erneuerbare Energien im hohen Maße zur Verfügung stehen. ${ }^{64}$ Prinzipiell kann ein effizientes und marktreifes Verfahren zur synthetischen Erzeugung von N-Düngern ohne Einsatz fossiler Brennstoffe durchaus Bestandteil einer zukunftsfähigen Land- und Düngewirtschaft sein - vorerst würde durch die Verknappung fossiler Brennstoffe aber auch die Produktion synthetischer NDüngemittel begrenzt. Dies reizt zunächst eine effiziente, standort- und kulturangepasste Düngung an, aber auch den Umstieg auf alternative Düngemittel wie organische Dünger und Rezyklate.

Da ein Großteil der mineralischen Düngemittel als Mehrnährstoffdünger verkauft wird, hätte eine Verknappung synthetischer N-Dünger letztlich auch Auswirkungen auf den Absatz der P-Dünger. Grundsätzlich könnte der P-Bedarf jedoch auch über Einnährstoffdünger gedeckt werden. Angesichts der Notwendigkeit, den Pflanzen sowohl P als auch N (sowie weitere Nährstoffe wie Kalium) zuzuführen, scheint es aber auch wahrscheinlich, dass stärker auf die Düngeeffizienz geachtet und vermehrt P- und N-haltige organische Dünger und Recycling-Produkte eingesetzt werden.

Zudem liegt mit dem reformierten Emissionshandel auch eine verstärkte Nutzung von Leguminosen für die N-Versorgung der Pflanzen nahe. Erfolgt der Leguminosenanbau im Rahmen vielgliedriger Fruchtfolgen, wirkt dies auch positiv auf die Effizienz der P-Düngung im Sinne einer verbesserten P-Aufnahme durch die Pflanzen und einer verstärkten P-Mobilisierung im Boden, u. a. durch einen gelockerten Boden, eine tiefe Durchwurzelung sowie aufgrund der Kopplung des mikrobiellen N- und P- Umsatzes. ${ }^{65}$

Geht man davon aus, dass weniger synthetische N-Dünger verfügbar wären und rohphosphathaltige Düngemittel teurer würden, würde dies wahrscheinlich auch den reinen Pflanzenanbau, insbesondere den reinen Futtermittelanbau, erschweren. Bestenfalls könnte eine stärkere Kopplung von Tierhaltung und Ackerbau angeregt werden, da die Abhängigkeit der Landwirtschaftsbetriebe von organischen Düngern erhöht wird. Dies gilt insbesondere dann, wenn die organischen Düngemittel im Zuge sinkender Tierzahlen (zum Emissionshandel für tierische Produkte im nächsten Unterkapitel) nur eingeschränkt verfügbar wären und weite Transportstrecken für organische Dünger aufgrund erhöhter Energiekosten ebenfalls vermieden

52) $\mathrm{Zu}$ den Einflussfaktoren auf die P-Preise insbesondere Mew/ Steiner/Geissler, Sustainability 2018, 1, 9 ff. Ausführlicher zu den Auswirkungen eines Primärenergie-Emissionshandels auf $\mathrm{P}$ siehe Garske, Instrumente (Fn. 7), Kapitel 5.3.2.

53) Index Mundi, Rock Phosphate Monthly Price, 1990-2020, Stand 20.2.2021, abrufbar unter https://www.indexmundi.com/commodities $/$ ?commodity $=$ rock-phosphate\&months $=360 ; \quad$ Rosema rin/Ekane, Nutr Cycl Agroecosyst 2016, 265, 268; Köhn/Zimmer/ Leinweber, Mechan Econ Regul 2017, 6, 15.

54) Khabarov/Obersteiner, Front in Nutr 2017, 1, 3.

55) $U B A$, Ökobilanzieller Vergleich der P-Rückgewinnung aus dem Abwasserstrom mit der Düngemittelproduktion aus Rohphosphaten unter Einbeziehung von Umweltfolgeschäden und deren Vermeidung, 2019, S. 24 und S. $56 \mathrm{ff}$.

56) Brentrup/Küsters, Energiebilanz der Erzeugung und Verwendung von mineralischen Düngemitteln - Stand und Perspektiven, in: KBTL (Hrsg.), Energieeffiziente Landwirtschaft 2008, 56, 60 ff.; UBA, P-Rückgewinnung (Fn. 55), Kapitel 3 und S. 78; Ramírez/ Worrell, Res Conserv Rec 2006, 75, 81.

57) $U B A$, P-Rückgewinnung (Fn. 55), S. 24 f. und S. 56.

58) KTBL (Hrsg.): Energieeffiziente Landwirtschaft, 2008

59) Kyriakou/Garagounis/Vourros/Vasileiou/Stonkides, Joule 2020, $142 \mathrm{ff}$; Razon, Environ Prog Sustainable Energy 2018, $618 \mathrm{ff}$. Sutton u. a., Nutrient World (Fn. 21), S. 8 und S. 16; Ahlgren/Bernesson/Nordberg/Hansson, Bioresour Technol 2010, 7181.

60) Die Energieverfügbarkeit könnte sich höchstens in einigen Dekaden ändern, wenn die EU und weitere Staaten das Ziel erreichen, Fusionsenergie breitflächig für die Energieversorgung zu nutzen.

61) Kyriakou/Garagounis/Vourros/Vasileiou/Stoukides, Joule 2020, $142 \mathrm{ff}$; Razon, Environ Prog Sustainable Energy 2018, $618 \mathrm{ff}$.; Sutton u. a., Nutrient World (Fn. 21), S. 8 und S. 16; Ahlgren/Bernesson/Nordberg/Hansson, Bioresour Technol 2010, 7181; Philibert, Producing industrial hydrogen from renewable energy, 2017; Goucher u.a., Nature Plants 2017, 1, 4; ISPT, Power to Ammonia. Feasibility study for the value chains and business cases to produce $\mathrm{CO}_{2}$-free ammonia suitable for various market applications, 2017, Chapter 2.

62) Bundesregierung Deutschland, Die nationale Wasserstoffstrategie 2020.

63) European Commission, $\operatorname{COM}(2020) 301$ final, 8.7.2020.

64) Philibert, Hydrogen (Fn. 61); ISPT, Power (Fn. 61), Chapter 2.

65) Grafe u.a., Environ Microbiol Reports 2018, $320 \mathrm{ff}$; Eichler-Löbermann/Köhne/Kowalski/Schnug, J Plant Nutr 2008, 659, 671; Bauke u. a., Plant Soil 2017, 67, 68. 
werden. Andererseits ließen sich organische Dünger besser transportieren, wenn Flüssig- und Festphase getrennt würden und grundsätzlich ist auch die Deckung der notwendigen Transportenergie durch erneuerbare Energien denkbar, wenngleich auch hier mit einer Steigerung der Transportkosten zu rechnen sein dürfte, was den regionalen Einsatz der organischen Dünger verhältnismäßig attraktiver macht. Dies gilt auch für den Transport von Futtermitteln, welcher durch die Verteuerung fossiler Brennstoffe erschwert werden dürfte, insbesondere über weitere Strecken wie etwa von Lateinamerika in die EU. Insofern würden auch P-Importe durch Futtermittelimporte unattraktiver. Durch steigende Rohphosphatpreise würden zudem auch P-Zusätze für Futtermittel teurer, was eine erhöhte P-Effizienz der Tierfütterung anregt.

Eine verstärkt organische Düngung könnte auch die notwendigen Importe mineralischer P-Dünger verringern, zumal auch diese wie erläutert teurer würden. Ein vollständiger Stopp der Importe von bergbaulich gewonnenem $\mathrm{P}$ würde allerdings nicht zielsicher durch die Verknappung fossiler Brennstoffe erreicht und auch auf die Qualität der importierten P-Düngemittel, insbesondere hinsichtlich ihrer Kontamination mit $\mathrm{Cd}$ und $\mathrm{U}$, hätte die Regulierung keinen Einfluss.

Neben der organischen Düngung würde auch das P-Recycling im Vergleich zur Nutzung bergbaulich gewonnener P-Düngern wettbewerblich bessergestellt. Insbesondere würden P-Rückgewinnungsverfahren mit niedrigem Energiebedarf bevorteilt, wobei auch hier letztlich entscheidend ist, dass die für die Rückgewinnung notwendige Energie mit Hilfe erneuerbarer Energieträger erzeugt wird.

Darüber hinaus würde der Ökolandbau mit seinem geringeren Einsatz fossiler Energie und dem Verzicht auf Mineraldünger durch die Verknappung fossiler Brennstoffe bevorteilt. Durch den Ökolandbau, insbesondere auch durch die in Art. 5 lit. g) VO (EG) Nr. 834/2007 i. V.m. Art. 14 Abs. 1 lit.d) Nr.i) VO (EG) Nr. 834/2007 vorgesehene Flächenbindung, würden weitere Vorteile für die Umweltmedien erzielt, ${ }^{66}$ zumal auch hierdurch die Futtermittelimporte reduziert würden.

Mit Blick auf die Tierhaltung erreicht ein Cap für fossile Brennstoffe trotz Preissteigerungen und trotz gegebenenfalls verstärkter Kopplung von Tierhaltung und Ackerbau voraussichtlich jedoch keine ausreichende Wirkung. Zwar würde der Primärenergie-Emissionshandel wie beschrieben verschiedene Preissteigerungen einschließlich der Verteuerung von Futtermitteln auslösen, doch insbesondere die Emissionen der Tierhaltung, vor allem die Methanemissionen der Wiederkäuer, würden nicht durch einen solchen Emissionshandel erfasst. Zudem würden durch die Begrenzung fossiler Brennstoffe die regional hohen Viehdichten und dementsprechend auch die Nährstoff-Hot-Spots nicht zielsicher vermieden und damit ein Kernproblem des PEinsatzes nicht adressiert. Auch auf die weiteren Probleme der Tierhaltung, etwa die Flächeninanspruchnahme und damit die biodiversitätsgefährdenden Landnutzungsänderungen, würde keine ausreichende Wirkung entfaltet. Insbesondere wäre nicht sichergestellt, dass die verfügbaren Flächen beispielsweise für die Gülleausbringung, nicht aber für den Futtermittelanbau genutzt werden, selbst wenn verstärkt Leguminosen in die Fruchtfolge eingebaut würden. Stattdessen könnte die Ernte sogar zunehmend zur Energiegewinnung in Biogasanlagen eingesetzt werden, was sich mit Blick auf die begrenzte Verfügbarkeit fruchtbarer Böden als kritisch erweist. Um Verlagerungseffekte von Umweltproblem zu Umweltproblem im Sinne eines verstärkten Drucks auf die Flächen aufgrund eines ausgeweiteten Energiepflanzenanbaus zu vermeiden, bedürfte es einer zusätzlichen Regulierung. ${ }^{67}$ Ebenso könnte die Gülle in Biogasanlagen mitvergoren statt für die Düngung genutzt werden. Es wäre somit eine zusätzliche Regulierung der Tierhaltung nötig, um einerseits regionale Nährstoffüber- schüsse $\mathrm{zu}$ vermeiden und um andererseits die Treibhausgasemissionen der Tierhaltung entsprechend der $1,5^{\circ} \mathrm{C}$ Grenze zu begrenzen und die weiteren Umweltfolgen der Tierhaltung einschließlich der Folgen des ausgedehnten Futtermittelanbaus einzudämmen.

\subsection{Emissionshandel für tierische Produkte}

Die Landwirtschaft ist mit einem Anteil von rund 18\% des globalen Treibhausgasstoßes beziehungsweise rund $10 \%$ des europäischen und $13 \%$ des deutschen Treibhausgasausstoßes enorm klimarelevant, wobei der Tierhaltungssektor (einschließlich der Lagerung und Ausbringung von Wirtschaftsdüngern) über $60 \%$ dieser Emissionen verantwortet. ${ }^{68}$ Gleichzeitig ist die Tierhaltung wesentlicher Treiber von Landnutzungsänderungen für den Futtermittelanbau, welcher oftmals unter hohem P- (und N-)Düngemittelund Pestizideinsatz in Monokultur erfolgt und dementsprechend biodiversitätsschädlich ist. Dem Tierhaltungssektor wird ein Beitrag von rund drei Vierteln am landwirtschaftlich verursachten Biodiversitätsverlust zugeschrieben. ${ }^{69}$

Mit Blick auf das Ziel, in ein bis zwei Dekaden Nullemissionen zu erreichen, müssten im Landwirtschaftssektor vermeidbare Emissionen komplett vermieden werden, etwa die $\mathrm{CO}_{2}$-Emissionen des (Transport-)Energieeinsatzes und der Düngemittelproduktion, die ohnehin durch den Primärenergie-Emissionshandel adressiert würden. Andere Emissionen müssten möglichst weit reduziert werden, insbesondere $\mathrm{CH}_{4}$ und $\mathrm{N}_{2} \mathrm{O}$ aus der Tierhaltung, den Böden und der Düngung. Verbleibende Emissionen müssten über Kohlenstoffsequestrierung, etwa durch Aufforstungsmaßnahmen oder die Erhöhung der Kohlenstoffspeicherung landwirtschaftlich genutzter Böden, ausgeglichen werden. ${ }^{70}$ Die Klimarahmenkonvention, auf der das ParisAbkommen basiert, deutet sogar auf die Landwirtschaft als Treibhausgassenke hin (Art. 4 Abs. 1 lit. d UNFCCC).

Abgesehen von den vermeidbaren und bereits mit dem Primärenergie-Emissionshandel adressierten Emissionen aus dem Energieeinsatz sind es insbesondere die Tierhaltungsemissionen, die es zu reduzieren gilt, da diese einen Großteil der landwirtschaftlichen Treibhausgasemissionen ausmachen und die Tierhaltung gleichzeitig wesentlicher Verursacher weiterer Umweltprobleme ist. Zugleich steht die Tierhaltung im Zentrum der P-Problematik. Bezogen auf das Klima bedeutet dies, dass die Treibhausgasemissionen der Tierhaltung einer Emissionsobergrenze unterlie-

66) Siehe Kapitel 3 zur Begrenzung externer Produktionsmittel einschließlich fossiler Energie im Ökolandbau sowie Boone/RoldánRuiz/Van Linden/Muylle/Dewulf, Sci Total Environ 2019, $1 \mathrm{ff}$.; Schader/Stolze/Gattinger, Environmental performance of organic farming, in: Boye/Arcand (Hrsg.), Green Technologies in Food Production and Processing 2012, $183 \mathrm{ff}$.

67) Ausführlich dazu Hennig, Landnutzung (Fn. 51). Siehe auch Ekardt/Wieding/Garske/Stubenrauch, CCLR 2018, 216 ff.

68) FAO, Tackling climate change through livestock - A global assessment of emissions and mitigation opportunities, 2013 , S. 15 ff.; UBA, Daten zur Umwelt, 2018, S.34ff.; Lóránt/Allen, Net-zero agriculture in 2050: how to get there? 2019, S. $13 \mathrm{f}$; Leip u. a., Environ Res Lett 2015, 1, 2f.; Goucher/Bruce/Caeron/ Koh/Horton, Nature Plants 2017, 1 ff. Die EU-Kommission rechnet dem Tierhaltungssektor sogar $70 \%$ der Agrar-Treibhausgasemissionen zu, vgl. European Commission, COM(2020) 381 final, 20.5.2020, S. 8 .

69) Leip u.a., Environ Res Lett 2015, 1, 6 ff.; Bowles/Alexander/Hadjikakou, Ecol Econ 2019, 128 ff.; Van Zanten u. a., Global Change Biol 2018, $1 \mathrm{ff}$.

70) Allen/Lóránt, Agriculture in a $1.5^{\circ} \mathrm{C}$ world 2018; Lóránt/Allen, Net-zero (Fn. 68), S. 7; Rockström u. a., Science 2017, $1269 \mathrm{ff}$; Ekardt/Jacobs/Stubenrauch/Garske, Land 2020, $1 \mathrm{ff}$.; Steffens u.a., PNAS 2018, 8252 ff.; IPCC, Land (Fn. 18); IPCC, 1, $5^{\circ} \mathrm{C}$ (Fn. 26), S.7; European Commission, COM(2018) 773 final, 28.11.2018, S.23. 
gen müssen, die im Einklang mit Art. 2 Abs. 1 PA steht. ${ }^{71}$ Bezogen auf die Biodiversitätserhaltungsziele gemäß CBD mangelt es hingegen an einer abbildbaren und messbaren Steuerungsgröße, an der ein ökonomisches Instrument ansetzen könnte. ${ }^{72}$ Da eine starke Reduktion der Tierzahlen unter Berücksichtigung des Klimaziels jedoch automatisch auch positiven Einfluss auf die Biodiversität hätte - weniger Tiere bedeuten auch weniger Futtermittel mit entsprechend weniger Landnutzung(-sänderungen), Pestizideinsatz etc. - ist nicht zwingend eine gesonderte Biodiversitätsregulierung nötig. Bezüglich der durch Nährstoffüberschüsse verursachten Eutrophierung als Gefährdungsfaktor für die Gewässerbiodiversität wird im Kapitel 4.1.1 mit der Flächenbindung ein effektives Instrument vorgeschlagen.

Grundsätzlich erweisen sich sowohl Steuern als auch Zertifikatmärkte für die Tierhaltung bzw. für die gesamte Landwirtschaft als administrativ sehr aufwendig, schon allein deshalb, weil eine große Anzahl landwirtschaftlicher Betriebe und klimarelevanter Einzelhandlungen betroffen wäre. Überschaubarer wäre hingegen die Anzahl der verarbeitenden Betriebe, das heißt etwa Schlachthöfe und Molkereien, bei Eiern auch die Erstabnehmer. Ein vielversprechender Ansatz ist daher ein produktbezogener Emissionshandel, der von pauschalisierten Emissionswerten pro Tier und pro Kilogramm Produkt ausgeht und so die Transaktionskosten im Vergleich zu anderen Ansätzen verhältnismäßig niedrig hält. ${ }^{73} \mathrm{Um}$ die Genauigkeit zu erhöhen, könnten statt EU-weiter (oder gar globaler) Emissionswerte auch regional bzw. national variierende Emissionswerte pro tierisches Produkt, welche mit Hilfe standardisierter Methoden gemäß IPCC-Richtlinien berechnet werden, heranzogen werden. Diese können zusätzlich auch nach verschiedenen Produktionsmethoden weiter differenzier werden. ${ }^{74}$ Die an die verarbeitenden Betriebe bzw. Erstabnehmer zu verteilenden Zertifikate wären dann in Übereinstimmung mit der $1,5^{\circ} \mathrm{C}$-Grenze schrittweise stark abzusenken, was die Tierhaltung insgesamt stark reduzieren würde. Innerhalb der Branche würden dann gegebenenfalls Verlagerungseffekte zulasten der emissionsintensiven Rinderhaltung hin zur Hühner- und Schweinhaltung auftreten. ${ }^{75} \mathrm{Da}$ der Primärenergie-Emissionshandel aber die Futtermittelimporte, insbesondere auch die Sojaimporte verteuert, würde auch die Hühner- und Schweinehaltung unattraktiver. Verlagerungseffekte ins Ausland würden wiederum durch Border Adjustments vermieden.

Während die starke Reduktion der Tierzahlen einen wesentlichen Beitrag zur Erreichung der Klima- und Biodiversitätsziele und zur Vermeidung weiterer Umweltprobleme wie etwa der Luft- und Bodenverschmutzung leisten würde, ${ }^{76}$ fällt die Bilanz der Wirkungen auf die P-bezogenen Probleme gemischt aus: Zunächst würden sinkende Tierzahlen einen geringeren Futtermittelbedarf bedeuten und damit die Importabhängigkeit von P in Form von Futtermitteln und Futtermittelzusätzen senken. Zudem würde für den geringeren Futtermittelanbau auch weniger P benötigt. Allerdings stünde bei drastisch gesunkenen Tierzahlen auch weniger $P$ aus organischen Düngemitteln zur Verfügung. Dadurch könnte der Weg zur Kreislaufwirtschaft, der eine Substitution rohphosphathaltiger Düngemittel vorsieht, erschwert werden. Es könnte sogar vermehrt auf rohphosphathaltige Düngemittel und damit auf eine endliche Ressource zurückgegriffen werden. ${ }^{77}$ Auch auf das Problem der Cd- und U-Belastung der rohphosphathaltigen Düngemittel würde keine Wirkung erzielt. Ebenso ausbleiben würde eine ausreichende Anreizwirkung zur Erhöhung der P-Düngeeffizienz sowie der P-Fütterungseffizienz. Insbesondere würde ein Emissionshandel für tierische Produkte nicht ausschließen, dass trotz absolut gesenkter Tieranzahl regional hohe Viehdichten bestehen und damit Nährstoffüberschüsse auftreten. Durch die Handelskomponente werden die Emissionszertifikate ohne Berücksichtigung der lokalen Gegebenheiten verteilt, so dass keine gezielte
Wirkung auf die Schließung betrieblicher bzw. regionaler Nährstoffkreisläufe - auch im Sinne einer stärkeren Kopplung von Tierhaltung und Ackerbau - entfaltet wird. Der Ökolandbau dürfte von einem Emissionshandel für tierische Produkte ebenfalls nicht profitieren. Zwar sind in der ökologischen Viehhaltung die flächen- bzw. tierbezogenen Emissionswerte niedriger, aufgrund teilweise niedrigerer Erträge überschreiten sie je Produkteinheit im Durchschnitt jedoch häufig die Werte der konventionellen Landwirtschaft, wenngleich die Emissionswerte stark schwanken und ökologisch bewirtschaftete Böden regelmäßig Vorteile bezüglich der Kohlenstoffspeicherung und der langfristigen Ertragssicherung trotz Klimawandelrisiken aufweisen. ${ }^{78}$

Insgesamt würde ein durch einen Emissionshandel verursachter höherer Preis für $\mathrm{CO}_{2}$ für emissions- und ressourcenintensive, tierische Nahrungsmittel ein veränderteres Konsumverhalten in Richtung einer klima- und biodiversitätsschonenderen, stärker pflanzlich basierten, zugleich weniger P-intensiven Ernährungsweise anregen. ${ }^{79}$ Trotz des doppelten Emissionshandels bliebe jedoch weiterer PSteuerungsbedarf bestehen.

\subsection{Phosphorspezifischer Ergänzungsbedarf}

Mit einem Primärenergie-Emissionshandel und einem Emissionshandel für tierische Produkte wurde eine Instrumentenkombination entworfen, die wie gesehen nicht nur zur Erreichung der Klima- und Biodiversitätsziele gemäß PA und CBD sowie zum Schutz der weiteren Umweltmedien beiträgt, sondern auch einen positiven Einfluss auf eine Reihe der P-bezogenen Problemlagen hat. Insbesondere werden durch Preissteigerungen für rohphosphathaltige Düngemittel Effizienzbemühungen sowie eine verstärkte Nutzung organischer und rezyklierter Düngemittel angeregt. Auch P-Importe durch die Einfuhr von Futtermittelimporten werden im Zuge steigender Transportkosten unattraktiver, zumal sinkende Tierzahlen den Futtermittelbedarf und damit auch den P-Düngebedarf für den Futtermittelanbau senken. Ungelöst bleibt jedoch die Problematik regionaler Nährstoff-Hotspots durch hohe Viehdichten. Diese kann relativ

71) Weishaupt/Ekardt/Garske/Stubenrauch/Wieding, Sustainability 2020, 1, $5 \mathrm{ff}$;; Weishaupt, Nachhaltigkeits-Governance (Fn. 26), S. 35; Ekardt/Jacobs/Stubenrauch/Garske, Land 2020, $1 \mathrm{ff}$.

72) Ekardt/Hennig, Ökonomische Instrumente und Bewertungen der Biodiversität. Lehren für den Naturschutz aus dem Klimaschutz? 2015.

73) Ausführlich zu den Vor- und Nachteilen eines solchen Ansatzes Weishaupt, Nachhaltigkeits-Governance (Fn. 26), S. 86; Weishaupt/Ekardt/Garske/Stubenrauch/Wieding, Sustainability 2020, 1 , 8 ff.; teilweise auch Gerber/Portet/Key/Steinfeld, Anim Int J Anim Biosci 2010, 393, 398; Wirsenius/Hedenus/Mohlin, Clim Change 2011, $160 \mathrm{ff}$.

74) IPCC, 2019 Refinement to the 2006 IPPC Guidelines for National Greenhouse Gas Inventories. Volume 4: Agriculture, Forestry and Other Land Use. Chapter 10: Emissions from Livestock and Manure Management, 2019; Weishaupt/Ekardt/Garske/Stubenrauch/Wieding, Sustainability 2020, 1, 12; Gerber/Portet/Key/ Steinfeld, Anim Int J Anim Biosci 2010, 393, $396 \mathrm{f}$.

75) Wirsenius/Hedenus/Mohlin, Clim Change 2011, 159, 173, Weishaupt/ Ekardt/Garske/Stubenrauch/Wieding, Sustainability 2020, 1, 13.

76) Siehe auch Leip u. a., Environ Res Letters 2015, 1, 6 ff.

77) Dies gilt auch für N. Zu sinkenden Wirtschaftsdüngermengen durch reduzierte Tierzahlen siehe auch Karsson/Röös, Land Use Pol 2019, 63, $70 \mathrm{f}$.

78) Smith/Kirk/Jones/Williams, Nature Communications 2019, $1 \mathrm{ff}$.; Schader/Stolze/Gattinger, Environmental performance of organic Farming, in: Boye/Arcand (Eds.), Green Technologies in Food Production and Processing, 2012, 183 ff.; Muller u. a., Nat Commun 2017, 1 ff.; Seufert/Ramankutty/Mayerhofer, Food Policy 2017, $10 \mathrm{ff}$; TEEB, Measuring what Matters in Agriculture and Food Systems, 2018, S. $37 \mathrm{ff}$.

79) Siehe auch Bowles/Alexander/Hadjikakou, Ecol Econ 2019, 128, 133. 
einfach durch eine Flächenbindung gelöst werden (dazu im Kapitel 3.3.1). Darüber hinaus löst die vorgeschlagene Instrumentenkombination nicht das Problem der Kritikalität von Rohphosphat bzw. rohphosphathaltiger Dünger sowie deren Cd- und U-Belastung. Und während der Emissionshandel für fossile Brennstoffe rohphosphathaltige P-Düngemittel verteuert und diese gegenüber organischen Düngern wettbewerblich schlechter stellt, verknappt der Emissionshandel für tierische Produkte die Menge vorhandener organischer Dünger. Das erhöht insgesamt den Druck auf P-Rezyklate, die dann einerseits mit Hilfe erneuerbarer Energie hergestellt werden müssten und andererseits den P-Bedarf weitgehend decken müssten - und das nicht nur auf nationaler bzw. europäischer Ebene, sondern auch im globalen Maßstab. Diesbezüglich stellen sich mehrere Fragen, die wie erwähnt teils von empirischen Befunden und dem nicht gänzlich geklärten normativen P-Ziel abhängig sind und insofern nur für bestimmte Szenarien bzw. unter Annahme bestimmter Voraussetzungen aufgelöst werden können (dazu im Kapitel 3.3.2).

\subsubsection{Vermeidung regionaler P-Überschüsse} durch eine Flächenbindung der Tierhaltung

Wie eingangs dargelegt, ist die Flächenbindung von Pflanzenbau und Tiererzeugung bislang nur für den Ökolandbau rechtlich verbindlich. So schreibt Art. 5 lit. g) VO (EG) Nr. 834/2007 i. V.m. Art. 14 Abs. 1 lit.d) Nr. i) VO (EG) Nr. 834/2007 vor, dass Futtermittel für die Tierhaltung hauptsächlich in dem Betrieb, in dem sie gehalten werden, oder in anderen ökologischen Betrieben im gleichen Gebiet erzeugt werden müssen. ${ }^{80}$ Die in der Tierhaltung anfallenden Wirtschaftsdünger wiederum können dann auf den betriebseigenen Flächen bzw. in der Region ausgebracht werden, wobei sich aus der Ausbringungsobergrenze für $\mathrm{N}$ aus Wirtschaftsdüngern in Höhe von $170 \mathrm{~kg} / \mathrm{ha}$ gemäß Anhang III Nr. 2 Nitratrichtlinie rund zwei Großvieheinheiten $(\mathrm{GV}) /$ ha landwirtschaftlicher Nutzfläche ergeben. ${ }^{81}$ Grundsätzlich gilt diese Ausbringungsobergrenze für $\mathrm{N}$ auch für konventionelle Betriebe. Aus ihr ergibt sich gewissermaßen eine Verbindung der Güllemenge und der Fläche. Sie erreicht allerdings keine zielsichere Begrenzung der Viehdichte bzw. der Bestandsgrößen, da rechtlich nicht ausgeschlossen wird, dass die über die Obergrenze hinaus anfallenden Wirtschaftsdünger verkauft werden oder Flächen zugekauft bzw. zugepachtet werden, auf denen aber keine tatsächliche Düngerausbringung erfolgt. ${ }^{82}$ Derartige Ausweichmaßnahmen müssten bei einer wirkungsvollen Flächenbindung rechtlich beschränkt werden. Hierfür kann etwa eine schlaggenaue Aufzeichnung tatsächlich ausgebrachter Düngemengen hilfreich sein, wie sie in Deutschland mit der Düngerechtsnovelle von 2017 eingeführt wurde. Ebenso müssten andere Schlupflöcher geschlossen werden, etwa durch eine Legaldefinition von „Region“, in der Futtermittel angebaut werden müssen und Wirtschaftsdünger ausgebracht werden dürfen, das heißt mit einer klaren Festlegung, wie weit die notwendigen Flächen von der Tierhaltungsanlage entfernt sein dürfen.

Zudem berücksichtigen ..sowohl die N-Obergrenze von $170 \mathrm{~kg} / \mathrm{ha}$ als auch die im Ökolandbau festgeschriebene maximale Viehdichte von zwei GV/ha keine standortspezifischen Eigenschaften wie Bodenart und Regionalklima. So ließe sich für sandige, durchlässige Standorte eine geringere Besatzobergrenze für Tiere empfehlen, während Standorte mit tonreicheren Böden gegebenenfalls auch mehr Großvieheinheiten zulassen würden. ${ }^{83}$ Eine standortangepasste Besatzobergrenze für Tiere erscheint daher empfehlenswert, wenngleich dies administrativ einen Mehraufwand bedeutet und $\mathrm{zu}$ unterschiedlichen Wettbewerbsbedingungen auch innerhalb eines Landes führen würde, die gegebenenfalls durch eine entsprechende Anpassung der Agrarsubventionen abgefangen werden könnten. Hinsichtlich der Agrarsubventionierung ist zudem festzuhalten, dass ein gewisses Steuerungspotenzial für die Flächenbindung auch darin liegt, die flächenunabhängig produzierende Tierhaltung von der Förderung im Rahmen der GAP auszuschließen. Noch zielsicherer wäre aber eine gesetzliche Verankerung der Flächenbindung, wofür sich neben dem Agrarrecht auch das Baurecht anbietet, so dass Gemeinden das Wachstum von Intensivtierhaltungsanlagen - beispielsweise auf zwei GV/ha oder weniger - begrenzen können. Eine flächenunabhängige Tierhaltung wäre dann nicht mehr genehmigungsfähig.

Eine für alle Betriebe in Deutschland bzw. in der EU verbindliche Flächenbindung würde nicht nur die Viehdichte pro Hektar verringern, sondern letztlich auch eine absolute Begrenzung der Tierhaltung in Deutschland bzw. in der EU bedeuten, da die mögliche Anzahl der gehaltenen Tiere von der begrenzt zur Verfügung stehenden landwirtschaftlichen Nutzfläche abhängig wäre. Wie in Kapitel 3.2 beschrieben, würde zusätzlich eine weitere Reduktion des Gesamtviehbestandes aufgrund des Emissionshandels für tierische Produkte stattfinden, um die Tieranzahl im Einklang mit dem Pariser Klimazieles zu begrenzen.

Eine Flächenbindung für alle Betriebe würde regionale P-Überschüsse durch hohe Viehdichten reduzieren und damit die Gefahr der Gewässereutrophierung verringern. Zudem würde sie die Verwendung von Zukauffuttermitteln senken und so auch den Import von P in Futtermitteln begrenzen. Damit würden auch die durch den Futtermittelimport ins Ausland verlagerten Gefährdungen von Böden, Gewässern und Biodiversität durch den Futtermittelanbau verringert. Stattdessen würde der (Zwischenfrucht-)Anbau heimischer Futtermittel, insbesondere auch der Leguminosenanbau, gestärkt. Um den Futtermittelanbau in weite Fruchtfolgen zu integrieren, wären gegebenenfalls flankierende Fördermaßnahmen und ordnungsrechtliche Restriktionen erforderlich, etwa bezüglich der Vermeidung von Grünlandumbruch im Zuge einer verstärkten Flächeninanspruchnahme für den Futtermittelanbau. Durch vielgliedrige Fruchtfolgen und durch die vermehrte Düngung mit organischen Düngern würde auch die P-Mobilisierung angeregt und die Erosionsgefahr vermindert, was positiv auf die Nutzungseffizienz von $\mathrm{P}$ wirken würde. Die stärkere Kopplung von Viehhaltung und Ackerbau ist zudem im Sinne der Kreislaufwirtschaft, da die Nährstoffversorgung der Pflanzen weitgehend durch das organische Material aus der Viehhaltung sichergestellt wird. Viehhaltende Ökolandbaubetriebe und Betriebe, die die Tierhaltung bereits flächengebunden betreiben, wären grundsätzlich von einer solchen Regelung bevorteilt und nicht von einer Umstellungspflicht betroffen. Betriebe, die ohne eigene Tierhaltung Futtermittel anbauen, würden je nach Ausgestaltung der Flächenbindung vor allem geographisch nah zu den viehhaltenden Betrieben in die Kreislaufwirtschaft einbezogen werden können. Reine Ackerbaubetriebe wären hingegen nicht von der Regelung betroffen und würden voraussichtlich weiter mit rohphosphathaltigen Düngemit-

80) Im Rahmen des deutschen BauGB meint dies, dass mindestens $50 \%$ der Futtermittel aus dem eigenen Betrieb stammen müssen. Siehe Deutscher Bundestag, WD 7 - 3000 - 066/17 v. 26. 5.2017, S. 7. Zur Berechnung des Futtermittelbedarfs und die Einbeziehung von Soja in diesen siehe auch VG München, Beschl. v. 6.6.2018-22 CS 18.1097. Ferner auch BMUB, Klimaschutzplan 2050, 2016, S. 66 zur Flächenbindung aus Klimaschutzgründen.

81) Wobei die Werte je nach Region und Methodik variieren und davon abhängen, wieviel $\mathrm{N}$ eine Großvieheinheit ausscheidet. Vgl. Velthof/Hue/Oenema, J Sci Food Agric 2015, 3004; Velthof, Report Task 1 of Methodological studies in the field of AgroEnvironmental Indicators. Lot 1 excretion factors, 2014.

82) Schulz, Good agricultural practice - fertilizer application in Germany in the scope of HELCOM recommendations, 2011, S. 2.

83) Siehe zu den unterschiedlichen (Dünge-)Anforderungen verschiedener Standorte Verhagen/Booltink/Bouma, Agric Syst 1995, 369, 375; Nett/Sradnick/Fuß/Flessa/Fink, Nutr Cycl Agroecosyst 2016, $217 \mathrm{ff}$. 
teln düngen, sofern P-Rezyklate nicht gesondert gefördert werden. ${ }^{84}$ Regulierungsbedarf verbleibt insofern insbesondere bezüglich der Verwendung rohphosphathaltiger Düngemittel und ihrer Kontamination mit Schadstoffen.

\subsubsection{Regulierung der Cadmium- und Uran-Gehalte in rohphosphathaltigen Düngemitteln und/oder deren Phasing Out}

Grundsätzlich ließe sich die Problematik der Schwermetallbelastung rohphosphathaltiger Düngemittel relativ einfach über ordnungsrechtliche Grenzwerte lösen. Allerdings konnte in der neuen Düngeproduktverordnung ${ }^{85}$ der EU von 2019 kein ambitionierter Grenzwert für Cd durchgesetzt werden, wodurch nicht zuletzt auch Steuerungspotenzial in Richtung der wettbewerblichen Besserstellung der in der Regel niedriger belasteten P-Rezyklate verschenkt wurde. ${ }^{86}$ Für U blieb die Grenzwertsetzung gänzlich aus.

Alternativ zu Grenzwerten wären prinzipiell auch Steuern auf die Schadstoffgehalte in Düngemitteln denkbar. ${ }^{87}$ Will man kurzfristige Gesundheitsgefahren vermeiden und kann man eine leicht vollziehbare Regelung anbieten hier auf Ebene der Düngemittelhersteller - erscheint der ordnungsrechtliche Weg vorzugswürdig. Letztlich würden beide Instrumente Marktvorteile für niedrig belastete Düngemittel einschließlich Rezyklaten und organischen Düngern bedeuten und Decadmierungsverfahren bzw. die Uranabscheidung anregen. Unabhängig von der Instrumentenwahl ist zudem zu beachten, dass auch P-Rezyklate und organische Düngemittel strengen Qualitäts- und Hygieneanforderungen unterliegen müssen.

Offen bleibt die Frage, ob der Einsatz rohphosphathaltiger Düngemittel, die nicht oder nur niedrig mit Schwermetallen belastet sind, überhaupt gewünscht ist. Dies ist, wie in der Einleitung angesprochen, eine normative Frage nach dem Umgang mit einer global begrenzten Ressource, für deren Reserven in Höhe von 69 Millionen Tonnen momentan eine statische Reichwerte von über 300 Jahren angenommen wird. ${ }^{88}$ Zudem sind die Vorkommen weltweit ungleich verteilt, wodurch sich auch Fragen der Verteilungsgerechtigkeit ergeben. $\mathrm{P}$ ist ein lebenswichtiges Element, das allen Menschen - global und dauerhaft - ausreichend zur Verfügung stehen muss. Nur so kann das Recht auf Nahrung gewährleistet werden. Während Art. 11 IPwskR explizit das Recht auf Nahrung verankert, impliziert das Recht auf Leben, Gesundheit, Freiheit und Sicherheit aus Art. 2, 3, 6 GRCh sowie Art. 2, 5 EMRK und Art. 2 GG nachhaltigkeitsbezogene Rechte wie den Zugang zu Nahrung, Wasser, sauberer Luft, einem stabilen Klima und intakten Ökosystemen sowie die Gewährung eines Existenzminimums. ${ }^{89}$ Grundsätzlich kann P aber auch aus organischen Düngemitteln und Recycling-Düngern zur Verfügung gestellt werden, so dass das Recht auf Nahrung prinzipiell auch ohne den Zugang zu rohphosphathaltigen Düngemitteln erfüllt werden kann. Die vordergründige Frage ist also, ob auch unter vollständigem Verzicht auf die begrenzten, rohphosphathaltigen Düngemittel ausreichende und sichere Nahrungsmittel produziert werden können.

Fraglich ist zunächst, wie hoch der P-Bedarf der deutschen bzw. europäischen bzw. globalen Landwirtschaft ist und inwiefern dieser P-Bedarf vollständig durch Recycling-Dünger und durch begrenzte Mengen organische Dünger gedeckt werden kann - und wenn ja, auf welcher Ebene: national, europäisch, international. Die Annahmen dazu, ob weiterhin rohphosphathaltige Düngemittel notwendig sind oder ob eine vollständige Substitution rohphosphathaltiger Düngemittel erreicht werden kann, können durchaus variieren, da der P-Bedarf der Landwirtschaft von verschiedenen Faktoren abhängig ist: Zunächst wird der P-Bedarf bestimmter Flächen von den vorhanden Boden-P-Gehalten und der Fähigkeit, die P-Gehalte im Boden zu mobilisieren, beeinflusst. Beides wiederum hängt von den Bodencharakteristika ab, die je nach Region mit ihren natürlichen Bedingungen sowie von den historischen und gegenwärtigen Bewirtschaftungsmethoden variieren. So binden eisen- und aluminium(hydr)oxidreiche Böden $P$ stark und erschweren die P-Mobilisierung, ${ }^{90}$ während beispielsweise Böden mit einem großen Vorrat an organischer Substanz positive Eigenschaften in Hinblick auf die Lösung und Mineralisierung von $\mathrm{P}$ besitzen. Zwischenfrüchte und eine diverse Fruchtfolge dienen wie erläutert der P-Mobilisierung und können den P-Bedarf nachfolgender Kulturen senken, zumal der P-Bedarf kulturabhängig ist und ohnehin weitere Möglichkeiten zur Erhöhung der P-Dünge- bzw. Aufnahmeeffizienz einschließlich der Präzisionsdüngung und der Nutzung von P-Aktivatoren bestehen. ${ }^{91}$ Hingegen können klimawandelbedingte Extremwetterereignisse wie Dürren den P-Bedarf vergröBern, während Starkniederschläge nach langer Trockenheit zu erhöhter Erosion und damit verbunden zu erhöhten P-Austrägen in die Gewässer führen, was im Endeffekt den P-Bedarf der betroffenen Flächen in der Folgezeit wieder erhöht. ${ }^{92}$ Darüber hinaus steht $\mathrm{P}$ aus verschiedenen Düngemitteln den Pflanzen innerhalb verschiedener Zeiträume zur Verfügung. So ist $\mathrm{P}$ aus organischen Düngemitteln im Gegensatz zu P aus Mineraldüngern erst langfristig verfügbar, so dass die kurzfristige P-Verfügbarkeit für die Pflanzen nicht unbedingt sichergestellt ist. Die P-Verfügbarkeit von Recycling-Düngern wiederum variiert und stellt somit einen weiteren Unsicherheitsfaktor für entsprechende Zukunftsprognosen dar. ${ }^{93}$ Die Beispiele zeigen, dass eine genaue Quantifizierung des P-Bedarfs für mehrere Jahre kaum möglich ist, insbesondere nicht auf globaler Ebene mit diversen Böden, Kulturen, Anbaumethoden usw.

84) Ausführlicher Garske, Instrumente (Fn. 7), Kapitel 5.2.3. Siehe auch Weishaupt/Ekardt/Garske/Stubenrauch/Wieding, Sustainability 2020, 1, 20.

85) Verordnung (EU) 2019/1009 des Europäischen Parlaments und des Rates vom 5.6.2019 mit Vorschriften für die Bereitstellung von EU-Düngeprodukten auf dem Markt und zur Änderung der Verordnungen (EG) Nr. 1069/2009 und (EG) Nr. 1107/2009 sowie zur Aufhebung der Verordnung (EG) Nr. 2003/2003, AB1. L 170, S. 1. Für den ursprünglichen Verordnungsentwurf mit schrittweise herabzusenkenden, strengeren Grenzwerten siehe European Commission, COM(2016) 157 final, S. 6 des Annex II

86) Ausführlicher zu Thematik Stubenrauch/Garske/Ekardt, Sustainability 2018, 1, $10 \mathrm{ff}$. und Ulrich, Sci Total Environ 2020, $541 \mathrm{ff}$.

87) Siehe am Beispiel von Schweden Watkins u.a. (Ed.), Capacity building, programmatic development and communication in the field of environmental taxation and budgetary reform, 2017, S. 68; Bomans u. a., Adressing (Fn. 15) und Ecotec u. a., Environmental Taxes (Fn. 38), S. 135 f. Siehe ferner European Commission, IP/027788 v. 31.5.2002 zum zusätzlich zur schwedischen Steuer geltenden Cd-Grenzwert für P-Düngemittel.

88) USGS, Mineral Commodity Summaries, 2020, S. 123; Van Kauwenbergh/Stewart/Mikkelsen, Better Crops with Plant Food 2013, 18, 20; zur Thematik auch Ulrich/Frossard, Sci Total Environ 2014, $694 \mathrm{ff}$.

89) Zur grundrechtlichen Basis und zur Diskussion um das Recht auf ein Existenzminimum Ekardt, Sustainability (Fn. 25), Chapter 3.

90) Gypser/Hirsch/Schleicher/Freese, J Environ Sci 2018, $175 \mathrm{ff}$.; Negassa/Dultz/Schlichting/Leinweber, Soil Sci 2008, 587; Koch u.a., Geoderma 2018, 115 ff.; Macintosh u.a., Sci Total Environ 2019, $90 \mathrm{ff}$.

91) Siehe Kapitel 1 sowie Eichler-Löbermann/Köhne/Kowalski/Schnug, J Plant Nutr 2008, 659, 670f.; Ohm/Paulsen/Moos/Eichler-Löbermann, Agron Sustainable Dev 2017, 1,2; Grafe u.a., Environ Microbiol Reports 2018, $320 \mathrm{ff}$; Zhu/Li/Whelan, Sci Total Environ 2018, 522, 529; Bergkemper u. a., Environ Microbiol 2016, 1988 ; Bauke u. a., Plant Soil 2017, 67, 68; Mogollón/Beusen/van Grinsven/ Westhoek/Bouwman, Glob Environ Change 2018, 149, $152 \mathrm{ff}$.

92) Zimmer/Kahle/Baum, Agric Water Manage 2016, $21 \mathrm{ff}$; Schoumans/Bouraoui/Kabbe/Oenema/van Dijk, AMBIO 2015, 180, 187 f.; Sharpley u.a., AMBIO 2015, 163, 166; Jeppesen u. a., J Environ Qual 2009, $1930 \mathrm{ff}$.

93) Kratz/Schick/Shwiekh/Schnug, Journal für Kulturpflanzen 2014, 261, 267 ff.; Roy, Ecol Eng 2017, 213 ff. 
Für die europäische Ebene bzw. für Deutschland existieren hingegen verschiedene Studien zur Deckung des momentan bestehenden P-Bedarfs der Landwirtschaft durch organische Düngemittel und P-Rezyklate, wobei die Aussagen der Studien variieren, zumal alle Berechnungen auf bestimmten, teils divergierenden Grundannahmen über die notwendige Menge der zu produzierenden pflanzlichen und tierischen Nahrungsmittel, Futtermittel sowie Energie- und Rohstoffpflanzen und möglichen Einsparpotenzialen beruhen. So gehen Jedelhauser/Binder grundsätzlich davon aus, dass ein nachhaltigeres P-Management - die Nutzung von rückgewonnenem $\mathrm{P}$ inbegriffen - den P-Import in die europäischen Mitgliedstaaten einschließlich Deutschlands überflüssig machen könnte. ${ }^{94}$ Damit im Einklang konstatieren Schoumans u.a., dass es möglich ist, den europäischen P-Kreislauf vollständig zu schließen und ferner die Gewässerqualität in Europa zu erhöhen, wenn die bislang importierten, synthetischen P-Dünger durch organische Dünger und P-Rezyklate ersetzt werden. ${ }^{95}$ Auf Deutschland bezogen wird in einer Studie des Wissenschaftlichen Bereichs für Düngefragen am Bundesministerium für Landwirtschaft von 2016 darauf verwiesen, dass momentan ein Großteil des Nährstoffbedarfs bereits durch organische Dünger und organische Reststoffe gedeckt wird, insbesondere durch Wirtschaftsdünger und Gärreste. ${ }^{96} \mathrm{Al}$ lerdings bestehen regional große Unterschiede bezüglich der Mengen anfallender Wirtschaftsdünger und Reststoffe, was eine vollständige Deckung des Nährstoffbedarfs der deutschen Landwirtschaft durch diese Stoffe erschwert. ${ }^{97}$ Kratz/Schick/Shwiekh/Schnug wiederum legen mit ihren Berechnungen zum Potenzial der organischen und organisch-mineralischen P-Düngung in Deutschland aus dem Jahr 2014 dar, dass das P-Potenzial verschiedener Sekundärrohstoffe, insbesondere von Wirtschaftsdüngern, Klärschlämmen, Fleischknochenmehlen bzw. deren Aschen sowie Kompost, bei rund 431 000-67000 Tonnen P liegt und somit der Bedarf von 326000-458000 Tonnen P durch ein konsequentes Nährstoff-Recycling vollständig gedeckt werden könnte. ${ }^{98}$

Grundsätzlich zeigt sich durchaus ein großes Substitutionspotenzial für rohphosphathaltige Düngemittel. Ebenso existiert eine Reihe technischer Möglichkeiten für das PRecycling, insbesondere aus Klärschlamm. Allerdings ist ein Teil der Verfahren noch nicht marktreif oder hat sich bislang nicht auf dem Markt durchgesetzt, ${ }^{99}$ was die Bedeutung der organischen Düngung vergrößert. Hinsichtlich der Verwendung von Gülle werden in derartigen Studien der momentane Tierbestand und somit die gegenwärtig anfallenden Mengen von Wirtschaftsdüngern angenommen. Angesichts der für die Erreichung der Klima- und Biodiversitätsziele notwendigen starken Reduktion des Tierbestandes stellt sich die Frage, inwieweit auch mit einer wesentlich geringeren Menge an Wirtschaftsdüngern aus der Tierhaltung auf rohphosphathaltige Düngemittel verzichtet werden kann, ohne deshalb mit Ertragseinbußen rechnen zu müssen. Zusätzlich könnten Effizienzmaßnahmen in der Tierfütterung im Zuge steigender Rohphosphatpreise und dadurch steigender Preise für P-Futtermittelzusätze auch zur verringerten P-Ausscheidung der Tiere führen. Gleichzeitig sinkt wie beschrieben mit verringerter Tieranzahl auch der P-Bedarf für den Anbau von Futtermitteln sowie für P-Zusätze in Futtermitteln, wodurch zumindest teilweise ein Ausgleich geschaffen werden dürfte. Zudem werden P-Rezyklate bei steigenden Preisen für Rohphosphat durch den Primärenergie-Emissionshandel wettbewerblich bessergestellt, wodurch das sinkende Angebot von Wirtschaftsdüngern ebenfalls abgefedert wird. Denkbar wäre auch ein zusätzliches Instrument zur Förderung des Einsatzes von Recycling-Düngern, um diese schneller und günstiger auf dem Markt verfügbar zu machen.

Unter Umständen muss die Frage nach dem Einsatz rohphosphathaltiger Düngemittel auch je nach Region un- terschiedlich beantwortet werden. Orientiert man sich an dem gesetzten Ziel der EU, vom Import des als kritische Ressource anerkannten Rohphosphats unabhängig(er) zu werden, ${ }^{100}$ muss dafür sowohl ein Zeitrahmen als auch ein entsprechendes Instrumentarium für die EU-Ebene festgelegt werden. Erreichbar wäre ein Ausstieg aus der Nutzung von Rohphosphat bzw. rohphosphathaltigen Düngemitteln grundsätzlich sowohl ordnungsrechtlich über ein Verbot als auch über ökonomische Instrumente, insbesondere einen Zertifikathandel, der die Menge des verfügbaren Rohphosphats schrittweise auf null absenkt. Da sich die Rohphosphatvorkommen nahezu ausschließlich auf Regionen außerhalb Europas konzentrieren (ausgenommen sind die Vorkommen in Finnland), läge es dann nahe, dass ein Zertifikatehandel auf EU-Ebene mit entsprechenden Border Adjustments nicht den Abbau von Rohphosphat, sondern den Verkauf von rohphosphathaltigen Düngemitteln in der EU schrittweise verknappt. Denkbar wäre eine Orientierung am kürzlich von der EU-Kommission in der Farm-to-Fork-Strategie avisierten Ziel, laut dem der Düngemittelverbrauch bis 2030 um $20 \%$ gesenkt werden soll, um Nährstoffüberschüsse zu reduzieren. ${ }^{101} \mathrm{Während} \mathrm{die-}$ ses Ziel alle Düngemittel mit einschließt, könnte speziell für rohphosphathaltige Düngemittel auch für die folgenden Dekaden ein Reduktionsziel von jeweils $20 \%$ festgelegt werden, das heißt bis 2040 eine Reduktion um 40\%, bis 2050 um $60 \%$, bis 2060 um $80 \%$, um in 50 Jahren, im Jahr 2070, keine rohphosphathaltigen Dünger mehr auf den Binnenmarkt $\mathrm{zu}$ bringen. Möglich wären auch (strenge) Ausnahmen zur Sicherstellung des kurzfristigen P-Bedarfs der Pflanzen, wenn die Nährstoffversorgung sonst nicht gewährleistet werden kann, etwa aufgrund schwieriger Witterungsverhältnisse in Verbindung mit der langfristigeren Löslichkeit von P in Wirtschaftsdüngern oder einigen Recycling-Produkten. Grundsätzlich kann aber angesichts des großen Substitutionspotenzials von Rohphosphat, noch stärker ausschöpfbarer Effizienzmaßnahmen bei der Düngung und des verringerten P-Bedarfs für Futtermittel bei sinkenden Tierzahlen eine (nahezu) vollständige Kreislaufführung von $\mathrm{P}$ durchaus als anzustrebendes Ziel gesetzt werden. Wichtig sind dann die konsequente Rückgewinnung von $\mathrm{P}$ aus Abwasser- und Abfallströmen und die weitestgehende Vermeidung von Verlusten, unter anderem auch durch Landwirtschaftspraktiken, die den Boden schützen und seine Erosionsanfälligkeit verringern. All dies steht auch im Einklang mit wasserrechtlichen Qualitätszielen beispielsweise aus der EU-Wasserrahmenrichtlinie. ${ }^{102}$

Während bei einem vollständigen Verzicht auf rohphosphathaltige Düngemittel die Cd- und U-Belastung keine

94) Jedelhauser/Binder, Res Conserv Rec 2015, 294, 296.

95) Schoumans/Bouraoui/Kabbe/Oenema/van Dijk, AMBIO 2015, $180,188$.

96) Wissenschaftlicher Beirat für Düngungsfragen/Bundesministerium für Ernährung und Landwirtschaft, Anwendung von organischen Düngern und organischen Reststoffen in der Landwirtschaft, 2016, S. 3 und S. 6; VDLUFA, Mitteilungen 2/2016, S. 2.

97) Wissenschaftlicher Beirat für Düngungsfragen/ Bundesministerium für Ernährung und Landwirtschaft, Anwendung (Fn. 96), S. 3 und S. 6; Schulz, Mineral nutrient accounting in Germany - levels, methods, results and possible contribution to reduce eutrophication risks - an introduction, 2013, S. 6; bezogen auf Europa auch Smil, Annu Rev Energy Environ 2000, 53, 63.

98) Kratz/Schick/Shwiekh/Schnug, Journal für Kulturpflanzen 2014, $261 \mathrm{ff}$.

99) Roy, Ecol Eng 2017, 213 ff.; UBA, P-Rückgewinnung (Fn. 55).

100) European Commission, COM(2020) 474 final, 3.9.2020, S. 1 und S. 6.

101) European Commission, COM(2020) 381 final, 20.5.2020, S. 7.

102) Richtlinie 2000/60/EG des Europäischen Parlaments und des Rates vom 23.10.2000 zur Schaffung eines Ordnungsrahmens für Maßnahmen der Gemeinschaft im Bereich der Wasserpolitik, ABl. L 327, S. 1. 
Rolle mehr spielt, müsste in der Übergangszeit sowie für den Fall von Ausnahmenregelungen wie oben beschrieben ein Grenzwert für die Schadstoffbelastung dafür sorgen, dass Gefahren für Gesundheit und Umwelt durch zu hohe Kontamination abgewehrt werden. Dies gilt insbesondere auch dann, wenn kein vollständiger Ausstieg aus der Ressourcennutzung von Rohphosphat angestrebt wird. Die weltweiten Rohphosphat-Ressourcen müssten dann kontrolliert und langfristig unter Berücksichtigung einer - entlang noch zu findender normativer Maßstäbe - möglichst gerechten globalen Verteilung abgeschmolzen werden. Dennoch lohnt sich auch in diesem Falle ein verstärktes Recycling, um die begrenzten Ressourcen zu schonen und möglichst vielen nachfolgenden Generationen einen Zugang zu ihnen zu ermöglichen.

\section{Fazit und Ausblick}

Überlässt man einen großen Teil des Steuerungsbedarfs übergreifenden Instrumenten wie dem PrimärenergieEmissionshandel, dem Emissionshandel für tierische Produkte und der Flächenbindung, wird sowohl eine Reihe der (nicht nur agrarbedingten) Probleme für Klima, Biodiversität, Luft, Böden und Gewässer gelöst als auch ein wesentlicher Beitrag zur Verringerung der P-bezogenen Probleme geleistet. Sodann besteht indes jedenfalls noch Bedarf nach einer Regulierung der Schadstoffbelastung
P-haltiger Dünger, wofür sich Grenzwerte für die Cd- und U-Gehalte rohphosphathaltiger und weiterer P-Düngemittel anbieten, sowie hinsichtlich der Schonung der begrenzen Rohphosphat-Ressourcen. Inwiefern ein vollständiger Verzicht auf rohphosphathaltige Düngemittel möglich und geboten ist, wurde diskutiert, und auf entsprechende empirische und normative Unsicherheiten sowie den weiteren Forschungsbedarf aufmerksam gemacht. Grundsätzlich könnte mit Hilfe eines weiteren Zertifikatehandels erreicht werden, dass die Menge der auf den (europäischen) Markt gebrachten rohphosphathaltigen Düngemittel schrittweise auf null gesenkt wird, bis die vollständige Substitution durch kreislaufwirtschaftsfreundliche P-Dünger gelingt, wobei eingeschränkt auch Ausnahmen denkbar sind, um die P-Versorgung der Pflanzen sicherzustellen. Vorgestellt wurden die Lösungsansätze insbesondere für die europäische Ebene. Denkbar wären neben nationalen Lösungen auch globale Ansätze. Fragen wie jene nach den genauen PZielen und nach der empirischen Situation der Böden verschiedener Regionen und Naturräume, insbesondere dahingehend, welche P-Versorgung die Böden aufweisen und welche P-Mengen für welche Quantität an Nahrung tatsächlich zwingend benötigt werden, bleiben Gegenstände weiterer Diskussionen und wissenschaftlicher Analysen - in deren Ergebnis dann auch das Verhältnis von Transnationalität und Regionalität in der P-Governance weiter justiert werden muss.

\section{Tagungsbericht zur 3. Promovierendenkonferenz Umwelt und Recht, 24. und 25.9.2020}

\section{Fabian Iwanczik}

Die „Promovierendenkonferenz Umwelt und Recht“ (ProKUR) ist eine zweitägige Veranstaltung, auf welcher sich Promovierende, deren Themen umweltrechtliche Bezüge aufweisen, gegenseitig kennenlernen, vernetzen und diskutieren können. Das Stadium des Promotionsvorhabens spielt für die Teilnahme keine Rolle: Von einem bloßen Promotionsinteresse bis hin zu einem fast einreichungsreifen Manuskript sind Personen aus allen Stadien willkommen und vertreten. Niedrigschwellig können (Promotions-)Themen vorgestellt sowie methodische und organisatorische Fragen diskutiert werden. Die Teilnehmer geben gegenseitig Anmerkungen und Hilfestellungen, beispielsweise über Gliederungsentwürfe und Exposés, die verschiedenen Finanzierungsmöglichkeiten oder den Umgang mit Literaturverwaltungsprogrammen. Aber auch für aktuelle Fragen des Umweltrechts besteht ein Raum zur Diskussion. Ein solcher Austausch zwischen den Doktoranden erfolgt mit-

Fabian Iwanczik, Wissenschaftlicher Mitarbeiter und Doktorand im Rahmen des Kompetenznetzwerks ,Zukunftsherausforderungen des Umweltrechts“ (KomUR) von Prof. Dr. Dr. Durner LL.M., Rheinische Friedrich-Wilhelms-Universität Bonn,

Bonn, Deutschland unter schon innerhalb des Arbeitsalltags oder im Rahmen spezieller Veranstaltungen an einzelnen Instituten oder Universitäten. Die ProKUR bietet darüber hinaus einen Rahmen für einen ortsübergreifenden Austausch mit vielfältigen Gesprächspartnern von anderen Universitäten. Auch externe Doktoranden erhalten so eine einfache Möglichkeit mit anderen Doktoranden in Kontakt zu kommen.

Nach Leipzig (2018) und Bremen (2019) richtete dieses Jahr ein Organisationsteam aus Münster die nunmehr dritte Auflage der ProKUR aus. Wegen der Covid-19-Pandemie fand die ProKUR erstmalig als Online-Veranstaltung statt, gewissermaßen als ,eProKUR“. 51 Teilnehmer, vor allem aus Deutschland, aber auch aus Österreich, den Niederlanden und Brasilien, schalteten sich aus ihren Büros, Küchen, Arbeits- und Wohnzimmern zu.

Nach einer Begrüßung durch das Organisationsteam richtete Prof. Dr. Sabine Schlacke (Universität Münster) ein Grußwort an die Teilnehmer. Sie lobte das ,vielfältige Programm“, welches ,neben den medienwirksamen Themen wie Klimaschutz" auch weitere Herausforderungen des Umweltrechts behandeln würde. Ihr Wunsch, dass die Teilnehmer eine „konstruktive Atmosphäre“ und „ein miteinander, nicht gegeneinander" haben würden, sollte sich während der zwei Tage bewahrheiten. 